\title{
Perception of local orientation from shaded images
}

\author{
FRANK E. POLLICK, HIROSHI WATANABE, and MITSUO KAWATO \\ ATR Human Information Processing Research Laboratories, Kyoto, Japan
}

\begin{abstract}
The perception of local orientation from shaded images was examined. In Experiment 1, subjects viewed a boundaryless Gaussian hill and judged local orientation using both a gauge figure and a pointing method. One subject reported an internally consistent surface which was incompatible with the judged light-source direction and model used to generate the image. The remaining subjects reported a surface similar to the generating one, and analysis of their results indicated a contour of zero difference between response and generating slants. This contour of zero slant difference was explored in three subsequent experiments using the pointing technique. These experiments investigated possible influences of luminance artifact (Experiment 2), perception of global orientation (Experiment 3), and self-occluding contours (Experiment 4). All three of these experiments yielded results similar to those of Experiment 1, with distinct contours of zero slant difference. This contour was explored for relationships with the simulated slant of the generating surface and the differential structure of image intensity. This analysis indicated that the contour of zero slant difference was approximately a line of constant slant which shared large regions of adjacency to the zero crossings of the second directional derivative of image intensity.
\end{abstract}

Most studies of the perception of shape from shading conclude that shading is a weak cue to shape, one that is unable to reliably convey local shape and is easily dominated by other sources of visual information. Although these studies clearly illustrate failures of shape from shading, it is reasonable to question the overall conclusion that shading is incapable of providing accurate shape information. Specifically, one can ask whether recovery of shape from shading is uniformly poor over an entire shaded image, or whether certain regions produce reliable performance. To address this issue, we obtained judgments of local surface orientation over a large image region and related the pattern of these judgments to the information available in the image.

The fundamental problem of shape from shading, to infer shape from the pattern of image intensity, is complex; properties of the object (local surface orientation and reflectance), light source (position and spatial extent), and viewing geometry (orthographic or perspective) all interact to produce the image intensity. To deal with this complexity, most computational models make the assumption that reflectance is constant, shading is lambertian, and viewing is orthographic. This coincides with the situation of a pointlight source at infinity with image intensity related to the angle between the local surface normal and the light-source direction. Although this assumption of lambertian shading makes it possible to recover geometrical characteristics of

We thank Peter Giblin for his helpful suggestions and the Royal Society of London for generously funding his visit to our lab. We acknowledge The Geometry Center of The University of Minnesota for use of its software Geomview in the exploration of potential stimuli. This work was partially supported by an HFSP grant to M.K. Correspondence should be directed to F. E. Pollick, ATR Human Information Processing Research Laboratories, Kyoto, Japan (e-mail: fpollick@hip.atr.co.jp). the surface, it does not make it possible to obtain a unique shape interpretation for a local patch of image intensity. Thus, to find a unique interpretation of a shaded image, computational theories commonly incorporate additional constraints (Freeman, 1994; Ikeuchi \& Horn, 1981; Oliensis, 1991a, 199 lb), perform regularization (Horn, 1990), or find approximate solutions (Pentland, 1984).

Given the image of a lambertian shaded object, there are several properties of the structure of the surface which can be inferred from just the image intensity (Ferraro, 1994; Koenderink \& van Doorn, 1980; Yuille, 1989). For example, Koenderink and van Doorn (1980) showed that the structure of the isophotes in the image (lines of constant brightness) were determined by the parabolic lines on the surface (lines which have zero principal curvature in one direction), and that the extrema of intensity could be found on these parabolic lines. In a similar analysis, Yuille (1989) showed that zero crossings of the second derivative in the direction of the gradient of the image intensity occur near the extrema of curvature. Given that these results are independent of light-source direction and allow some relaxation of the lambertian shading assumption, they suggest that, in general, much of the descriptive geometry of an object is available from direct examination of the differential structure of the image intensity.

To obtain a 3-D metric interpretation, several shape-fromshading algorithms assume that at particular locations on the image there are additional constraints to the projected point's orientation or position. Two such locations are at the occluding contour and at such singular points as the points of maximal brightness (Koenderink \& van Doorn, 1993). At the occluding contour, it can be assumed that the direction of the surface normal is perpendicular to the viewing direction. This assumption was used by Ikeuchi 
and Horn (1981) as a boundary condition within a regularization framework, as well as by Hayakawa, Nishida, Wada, and Kawato (1994) in a scheme using forward and approximated inverse optics (Kawato, Hayakawa, \& Inui, 1993). For a given image, points of maximal brightness (the set of singular points) include the point that is closest to the light source. Oliensis (1991a, 1991b), in advancing the dynamic systems approach of Saxberg (1989), has indicated under what conditions the set of singular points provide a unique shape interpretation and when it only partially constrains the number of interpretations. He also demonstrated for this approach that the information at the occluding boundary is not influential in determining shape, that it is, in fact, boundary regions where shape is generally the least specified (Oliensis, 1991a).

Investigations of human perception of shape from shaded images have used judgments of local orientation (Koenderink, van Doorn, \& Kappers, 1992; Mamassian \& Kersten, in press; Mingolla \& Todd, 1986), absolute depth, as provided by a stereo probe (Bülthoff \& Mallot, 1988), relative depth (Todd \& Reichel, 1989), local surface geometry, as rated by a shape index (Erens, Kappers, \& Koenderink, 1993b), orientation of a local surface perturbation (Erens, 1993; Erens, Kappers, \& Koenderink, 1993c), and categorization of local surface type as elliptic or hyperbolic (Mamassian, Kersten, \& Knill, in press) as means whereby the perceived shape of a shaded stimulus is revealed.

Evaluation of the results of these studies from the viewpoint of consistency and accuracy does not lead to a consistent view of what shading provides. On the one hand, Koenderink et al. (1992), in addressing the issue of consistency, reported stable and coherent percepts of surface which varied qualitatively among subjects. (However, it is worth noting that Koenderink et al.'s stimulus did contain some texture information as well as self-occluding contours, and that this might have contributed to the consistency of responses.) On the other hand, experiments addressing the issue of accuracy have generally suggested that shading does not provide an accurate perception of shape. For example, Erens and his colleagues found that subjects could not categorize local shape (1993c) and that, even in the presence of global shape information, their performance in judging a local perturbation did not increase (1993a) past a somewhat low level. Mamassian et al. (in press) have indicated that, although there is a bias towards ellipticity, local shape can be reliably segregated into elliptic and hyperbolic regions, and that this ability is best around regions of occluding and attached shadow contours. Mingolla and Todd (1986) found a substantial trend for responded surfaces to appear flatter than the stimulus used to generate the shaded surface-that is, to "regress into the picture plane." This led Todd and Reichel (1989) to propose a model of shape from shading that primarily represented ordinal structure and other nonmetric properties. A similar tendency for "regression to the picture plane" was reported by Bülthoff and Mallot (1988) for shaded ellipsoids and by Mamassian and Kersten (in press) for a croissantshaped object.
The issue of accuracy in interpreting subjects' judgments of shape from shading is difficult. Due to the large number of factors that interact to produce a shading pattern, it is generally possible to find an infinite number of mathematically plausible solutions. Thus, although viewers of a shaded image typically make unique interpretations, choosing which of the infinite number of mathematically possible interpretations to compare against for an evaluation of accuracy is problematic. In this research we selected the surface used to generate the shading pattern as a standard for comparison. While this particular selection is arbitrary, it allows us to address the issue that reports of perceived shape, when compared with the generating surface, regress toward the picture plane (i.e., the difference between responded and generating slant is negative). Moreover, since the generating surface in many cases appears to be a popular interpretation of the pattern of image intensity, we can address the problem of what image information was used to obtain the interpretation if indeed we find that subjects are providing an interpretation similar to the generating surface.

In Experiment 1, we investigated reports of local surface orientation from a single shaded image of a borderless Gaussian hill. The results demonstrated subjects' abilities to report local surface orientation in the presence of only shading information, and indicated that, compared with the generating surface, there were coherent regions where reported slant was greater than that of the generating surface (positive slant differences) and less than that of the generating surface (negative slant differences), whose border produced a contour of zero slant difference. Subsequent experiments investigated whether such a contour of zero slant difference would be found under other conditions. These conditions included (1) the same Gaussian hill viewed at two different orientations under identical illumination, (2) a shaded Gaussian hill placed atop a textured box and viewed with stereo and perspective cues to depth, and (3) irregularly shaped blob surfaces with selfoccluding contours.

\section{EXPERIMENT 1}

In Experiment 1, we examined reports of local surface orientation from the shaded image of a Gaussian hill. Such a stimulus was chosen because it could be presented without self-occluding contours as a source of additional shape information. We obtained local orientation judgments using both the gauge figure technique of Koenderink et al. (1992; see also Pentland, 1982, and Stevens \& Brookes, 1987) and a pointing technique (Pollick, Nishida, Koike, \& Kawato, 1994). Both the consistency of the responses in forming a coherent, best fitting surface and the deviation from the generating surface (the surface used to generate the shaded image) were analyzed. The deviation of the responses from the generating surface was assessed by analyzing the relationship between the simulated slant and tilt of the generating surface and the responded slant and tilt. Slant and tilt were chosen to express orientation because of their rela- 


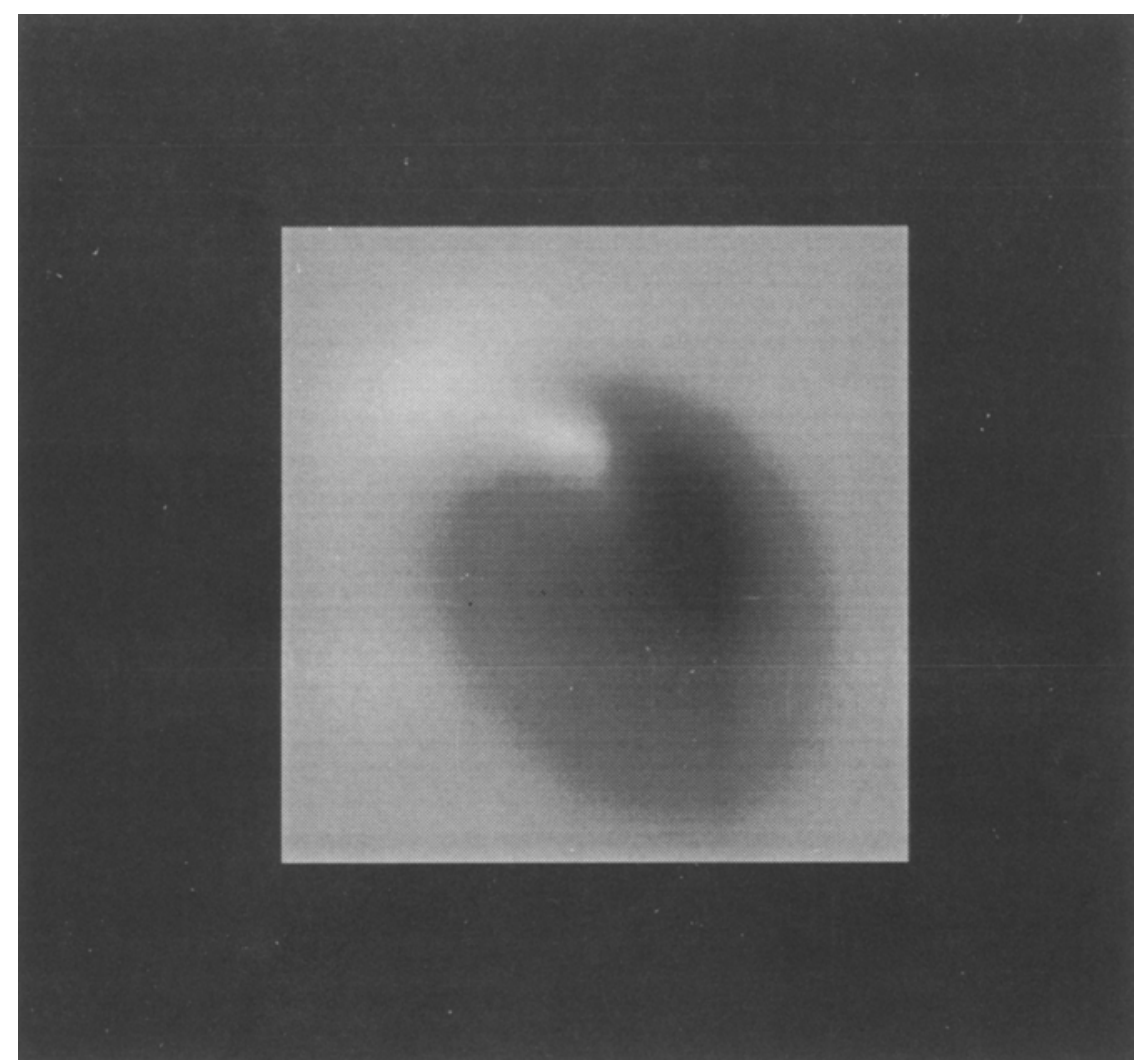

Figure 1. A reproduction of the stimulus used in Experiment 1. The grid of 196 probe points was centered approximately at the top of the hill of the generating surface and covered the central region.

tionship with representations of surface shape (Stevens, 1983a, 1983b). Because pilot studies with the shaded image of a Gaussian hill sometimes revealed best fitting surfaces that were qualitatively different from the generating surface, we obtained judgments of light-source direction and closest-point-to-the-observer to ascertain whether this qualitative difference could be accounted for by a mismatch between generating and reported light-source direction and if it was consistent with ordinal judgments of global structure.

\section{Method}

Subjects. There were 4 subjects, all of whom were naive as to the purpose of the experiment. The subjects were undergraduates at a local university who volunteered for the experiment and received payment for their participation. All subjects were right-handed and had normal stereo vision and visual acuity of at least $20 / 30$ in the eye used to view the stimuli.

Design. In the surface-recovery task, subjects judged local orientation at 196 locations in a single session, once with a pointing technique and once with a gauge figure technique. The order of the response methods was counterbalanced between the 4 subjects. For each session of the surface-recovery task, the subjects judged lightsource direction and the point on the image that appeared to be closest to them. In addition to these judgments, the subjects participated in a pretest and a posttest session in which they responded eight times to each location in a set of nine locations presented in a random order. The purpose of this pre-/posttest was to assess the variability of repeated measurements as well as to see if learning played a role in either of the response methods.
Stimuli and Apparatus. The stimulus of a shaded hill was generated by using orthographic projection and lambertian shading with a light source located at infinity in the direction $(-0.5,1,1)$ (to the seated subject, this direction is coming from behind, from the upper left). The shaded image of the surface was constructed by using a rayshooting technique to obtain preimage points on the surface and then calculating the surface normal and corresponding lambertian intensity. To avoid subjects' possible use of symmetry, the shape of the hill was made nonsymmetric (details of the generating hill are given in the Appendix). There were 196 points when the subjects responded to the local surface orientation. These 196 locations were organized in a $14 \times 14$ hexagonal array in the image, which subtended a width of $6.5^{\circ}$ and a height of $5.4^{\circ}$ (degrees of visual arc) and was centered approximately at the center of the projection of the hill (Figure 1).

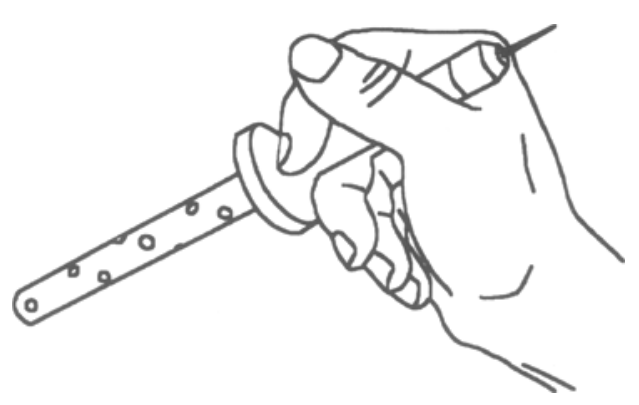

Figure 2. The wand as held by subjects to indicate local surface orientation. 

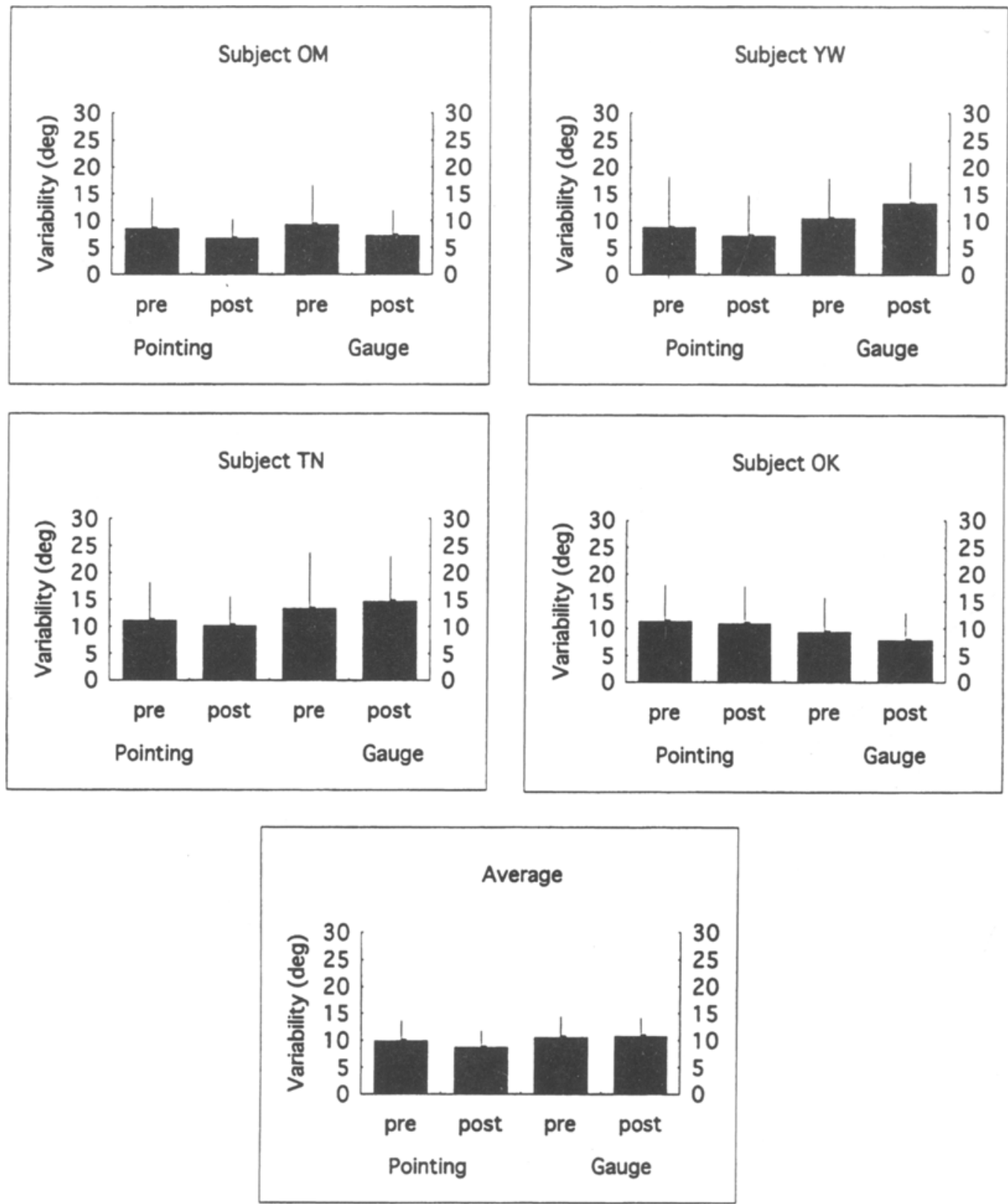

Figure 3. The variability of the responses about their centroids from the pre-/posttest portion of Experiment 1. The results of the pretest and the posttest for both the pointing and the gauge-figure methods are shown for the individual subjects as is their combined average.

The stimulus was presented on a Silicon Graphics IRIS Crimson/ Reality Engine with 1,280 × 1,024-pixel resolution. The subjects were seated and viewed the display from a distance of approximately $1 \mathrm{~m}$. An aperture was placed in front of the screen and limited the field of view on the display screen to a $12.4^{\circ}$ visual angle square. In addition, an occluder was placed approximately $82 \mathrm{~cm}$ from the display to restrict the view of the display to only the left eye. This configuration permitted a monocular view of the shaded image while enabling a binocular view of the digitizing wand used for the pointing responses.

The subjects responded to the surface orientation using two methods, one by pointing with a 3-D digitizing wand and the other by adjusting a mouse-operated gauge figure displayed on the screen. The 3-D digitizing wand (OPTOTRAK 25-marker digitizing probe, De- sign 2, Northern Digital) and how it was grasped is shown in Figure 2 . The wand was instrumented with 25 infrared light-emitting diodes, whose positions were measured with a $3-D$ position-analysis system (OPTOTRAK, Northern Digital), and the absolute orientation of the wand was calculated by using a technique based on quarternions (Horn, 1987). The subjects handled the wand with their right hands and were instructed to make the judgments with the near end of the wand located slightly below eye level. Note that the end of the wand facing the subjects appeared as a disk with a needle perpendicularly projecting from its center (quite similar in appearance to the gauge figure to be described next). The gauge figure simulated a ring (diameter $0.46^{\circ}$ of visual arc) with a perpendicular line (length $0.23^{\circ}$ of visual arc) projecting from its center, superimposed 


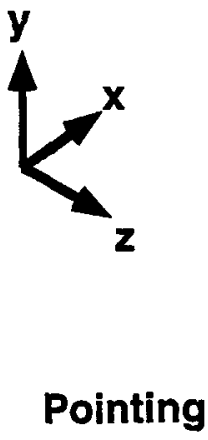

B

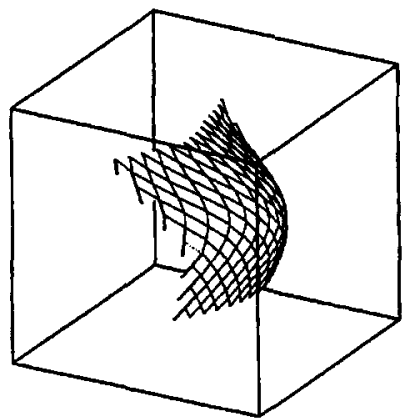

D

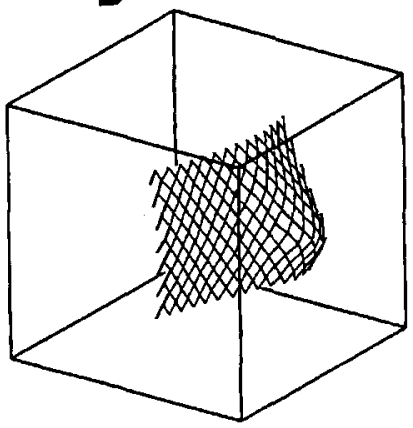

\section{Generating}

A

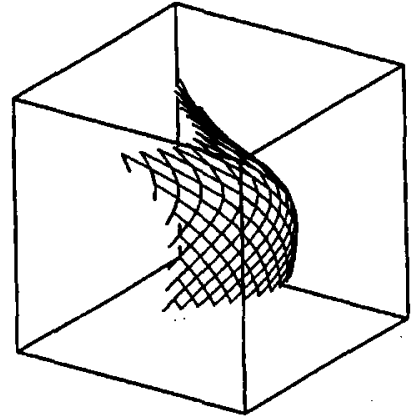

Gauge

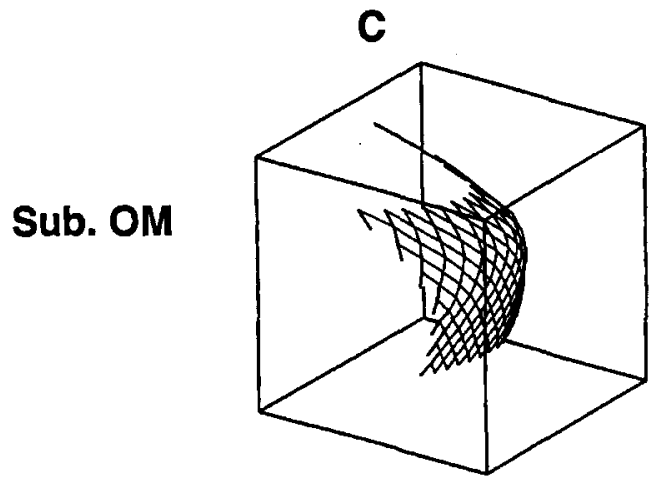

E

Sub. OK

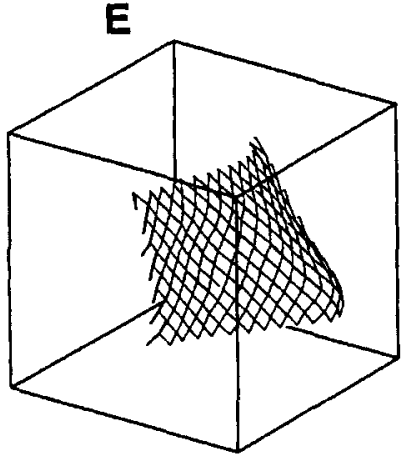

Figure 4. Examples of the best fitting surface to the 196 samples of surface orientation from Experiment 1. Shown are the recovered surfaces for the generating values $(A)$, the responses of Subject O.M. for pointing (B) and gauge (C), and the responses of Subject O.K. for pointing (D) and gauge (E).

on the surface of the shaded object. The range of simulated orientations of the gauge figure was $0^{\circ}-90^{\circ}$ slant and $0^{\circ}-360^{\circ}$ tilt. Vertical motion of the mouse controlled slant and horizontal motion of the mouse controlled tilt. When the gauge figure was properly adjusted, it appeared to sit on the surface being probed.

For the judgments of light-source direction, the subjects oriented either the gauge figure or the digitizing wand in a direction parallel to the perceived direction of illumination. For the judgments of the closest point, the subjects adjusted a cursor-controlled probe on the image until it matched the perceived location of the closest point.

Procedure. In a single session, the subjects used either the pointing or the gauge-figure technique. They performed the separate tasks in the following order: (1) pretest, (2) surface-recovery reports of 196 locations, (3) reports of light-source direction and closest point, and (4) posttest. Each session was self-paced, and the subjects took rests as needed within a session and a long rest (approximately $30 \mathrm{~min}$ ) before the posttest. At the beginning of the experiment, the subjects were instructed on how to respond to the perceived surface orientation and practiced several trials until they were comfortable with the response method.

\section{Results}

Pretest, posttest. The purpose of the pre- and posttests was to examine whether there was any difference between the gauge and pointing techniques as well as to examine the variability of repeated responses to the same point. Variability was defined as the average angular distance from the centroid of the responses to the actual responses, with the centroid obtained by calculating the average of the $x y z$ coordinates. The variability results, averaged across the nine locations, are shown in Figure 3. An analysis of variance (ANOVA) was performed on these variability mea- 
Table 1

Judgments of Light Source Direction and Closest Point From Experiment 1

\begin{tabular}{|c|c|c|c|c|c|c|c|}
\hline \multirow[b]{3}{*}{ Subject } & \multirow{3}{*}{$\begin{array}{l}\text { Response } \\
\text { Technique }\end{array}$} & & & \multicolumn{4}{|c|}{$\begin{array}{l}\text { Position of Closest Point } \\
\text { (Arbitrary Image Units) }\end{array}$} \\
\hline & & \multicolumn{2}{|c|}{$\begin{array}{l}\text { Light-Source } \\
\text { Direction }\end{array}$} & \multicolumn{2}{|c|}{$\begin{array}{l}\text { Ordinal } \\
\text { Judgment }\end{array}$} & \multicolumn{2}{|c|}{$\begin{array}{c}\text { Top of Best Fitting } \\
\text { Surface }\end{array}$} \\
\hline & & Slant & Tilt & $x$ & $y$ & $x$ & $y$ \\
\hline \multirow[t]{2}{*}{ T.N. } & Pointing & $47^{\circ}$ & $134^{\circ}$ & 0.22 & 0.35 & -0.40 & -0.09 \\
\hline & Gauge & $89^{\circ}$ & $142^{\circ}$ & 0.32 & 0.29 & -0.30 & 1.47 \\
\hline \multirow[t]{2}{*}{ Y.W. } & Pointing & $71^{\circ}$ & $140^{\circ}$ & 0.08 & 0.99 & 0.20 & 0.26 \\
\hline & Gauge & $60^{\circ}$ & $121^{\circ}$ & 0.14 & 0.89 & 0.20 & 0.60 \\
\hline \multirow[t]{2}{*}{ O.M. } & Pointing & $51^{\circ}$ & $132^{\circ}$ & 0.14 & 0.71 & 0.10 & 0.43 \\
\hline & Gauge & $70^{\circ}$ & $143^{\circ}$ & 0.11 & 0.83 & 0.00 & 0.95 \\
\hline \multirow[t]{2}{*}{ O.K. } & Pointing & $92^{\circ}$ & $35^{\circ}$ & 1.09 & -0.11 & 1.00 & -0.43 \\
\hline & Gauge & $46^{\circ}$ & $22^{\circ}$ & 1.04 & -0.53 & 1.10 & 0.09 \\
\hline $\begin{array}{l}\text { Averages for T.N., } \\
\text { Y.W., and O.M. }\end{array}$ & & $65^{\circ}$ & 136 & 017 & 0.68 & -0.03 & 0. \\
\hline Generating values & & $48^{\circ}$ & $117^{\circ}$ & 0.09 & 0.58 & 0.09 & 0.58 \\
\hline
\end{tabular}

surements to see if there was any effect of the type of response or the pre/post manipulation. The analysis showed no significant effects of either.

Best-fitting surface. The 196 surface-orientation judgments were converted into a best fitting surface using the procedure outlined by Koenderink et al. (1992). This procedure converted the 196 local orientations into least squares best estimates of the distances between the 533 pairs of adjacent points. Examples of the recovered surface for both the gauge and pointing techniques are shown in Figure 4 for 2 of the subjects. The recovered surfaces of the other 2 subjects were roughly similar to that of Subject O.M., with differences that fell into no consistent pattern.

The procedure for finding the best fitting surface also provided measures of the goodness of the fit. One such measure was whether individual triangular faces conformed to integrable surface patches (Koenderink et al., 1992). This measure was calculated, and the results of the gauge- and pointing-response techniques were compared; no significant differences were obtained. However, one interesting note was that although the shape recovered by Subject O.K. was different from that of the other subjects, there was no apparent difference among any of the subjects in how well individual patches conformed to an integrable surface.

Closest point and light-source direction. The judgments of closest point and light-source direction served to check that subjects' judgments of ordinal structure were consistent with the generating surface and that the reported light source direction was consistent with the generating light source. The judgment of closest point could also be compared with the closest point of the best fitting surface. This was useful in the case of subjects like Subject O.K., who reported a shape quite different from that of the generating surface. Table 1 shows the results of these judgments for the 4 subjects. It can be seen that, for both judgments, 3 of the subjects had responses which, on the average, were similar to the arrangement used to generate the stimulus. Subject O.K. made different judgments of both closest point and light-source direction, with the judgment
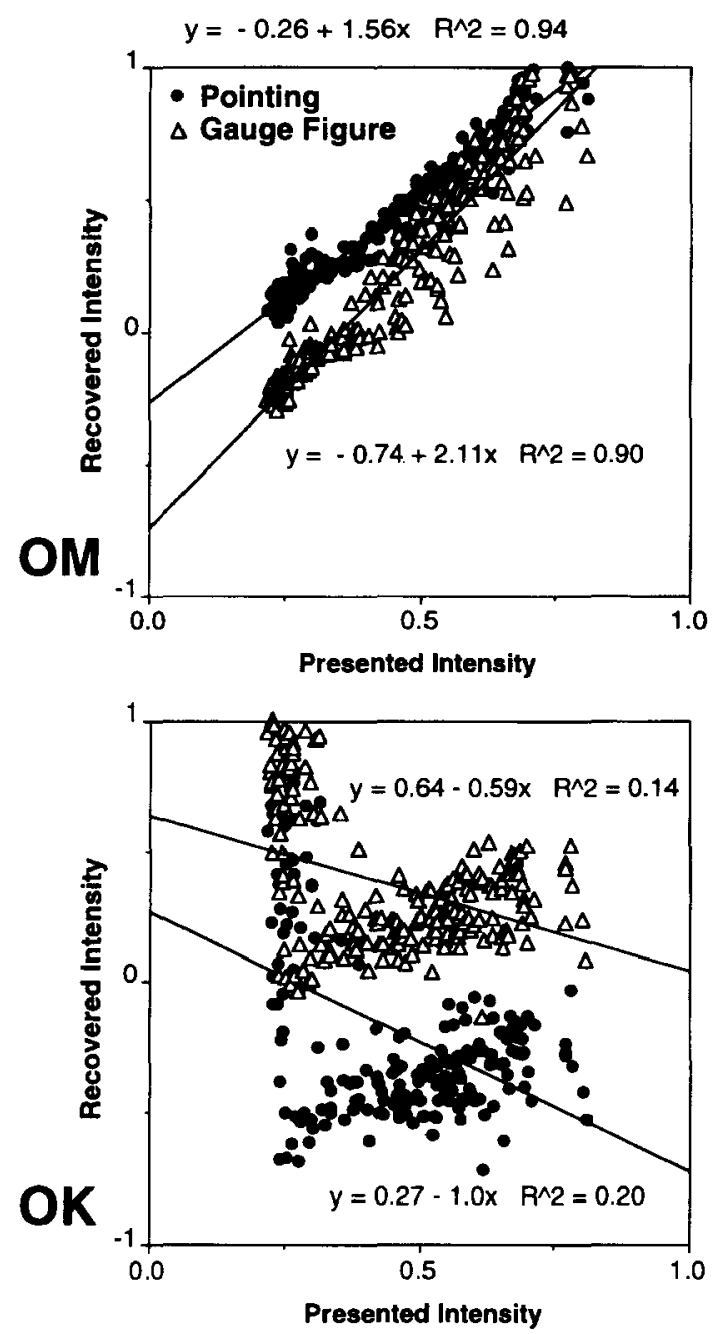

Figure 5. Plots of recovered image intensity versus presented image intensity from Experiment 1 for Subjects O.K. and O.M. Recovered image intensity was estimated from the dot product of reported light-source direction and normals of the best fit surface. Shown with the scatter of points are the values of the linear regressions. 


\section{Pointing}

YW

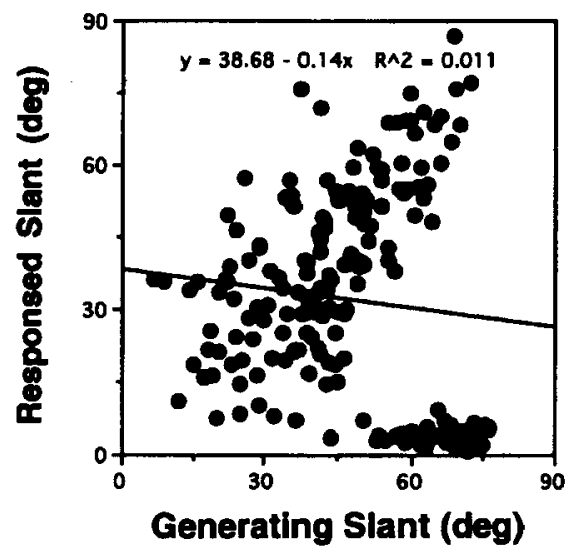

OM

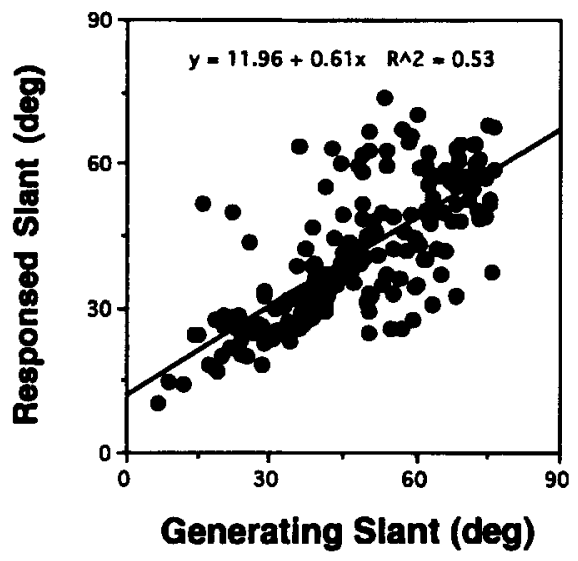

TN

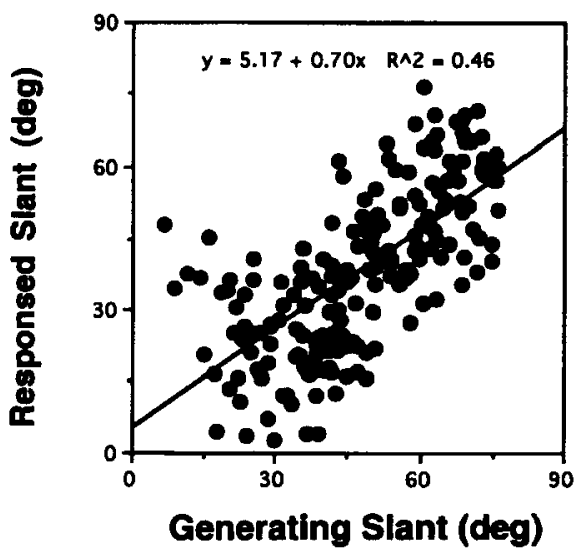

Gauge Figure
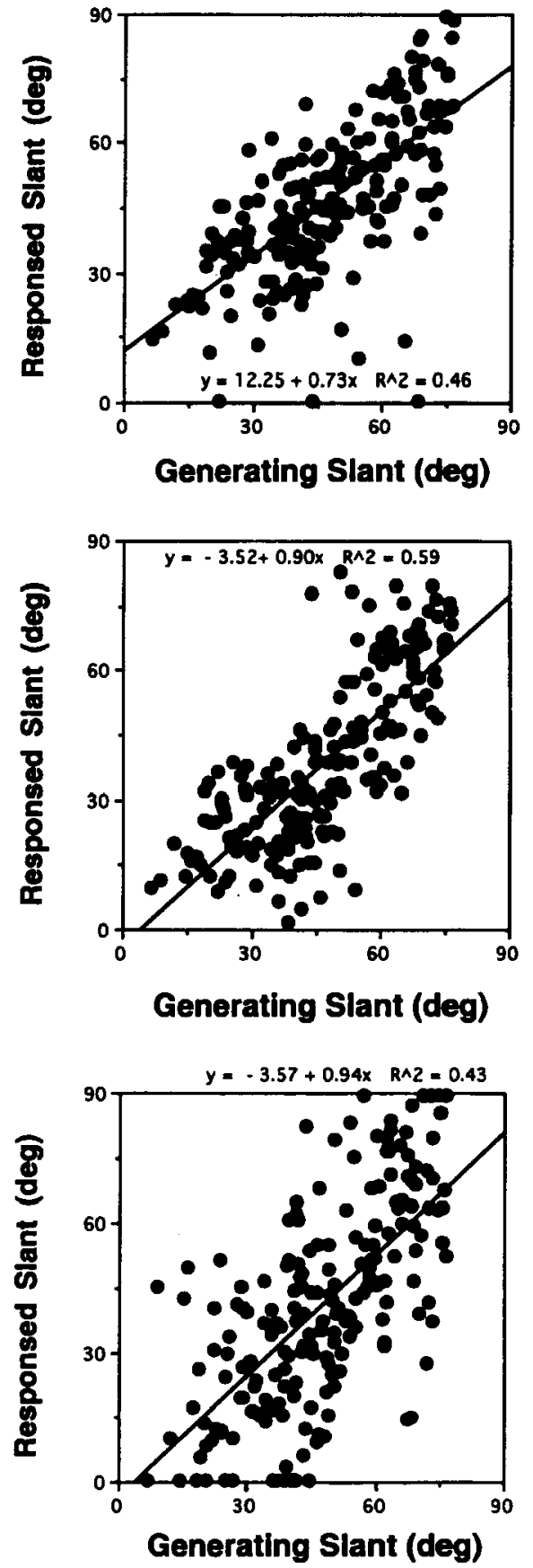

Figure 6A. Plots of slant versus generating slant from Experiment 1 along with linear regressions, for individual subjects (excluding Subject O.K.). 
Pointing

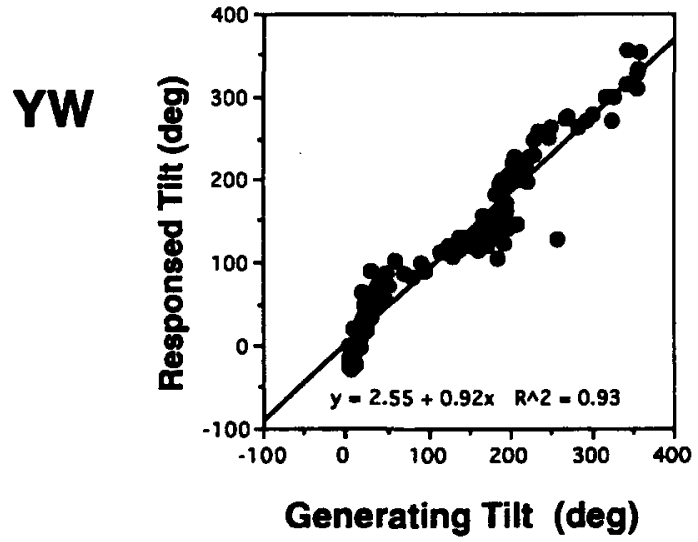

OM
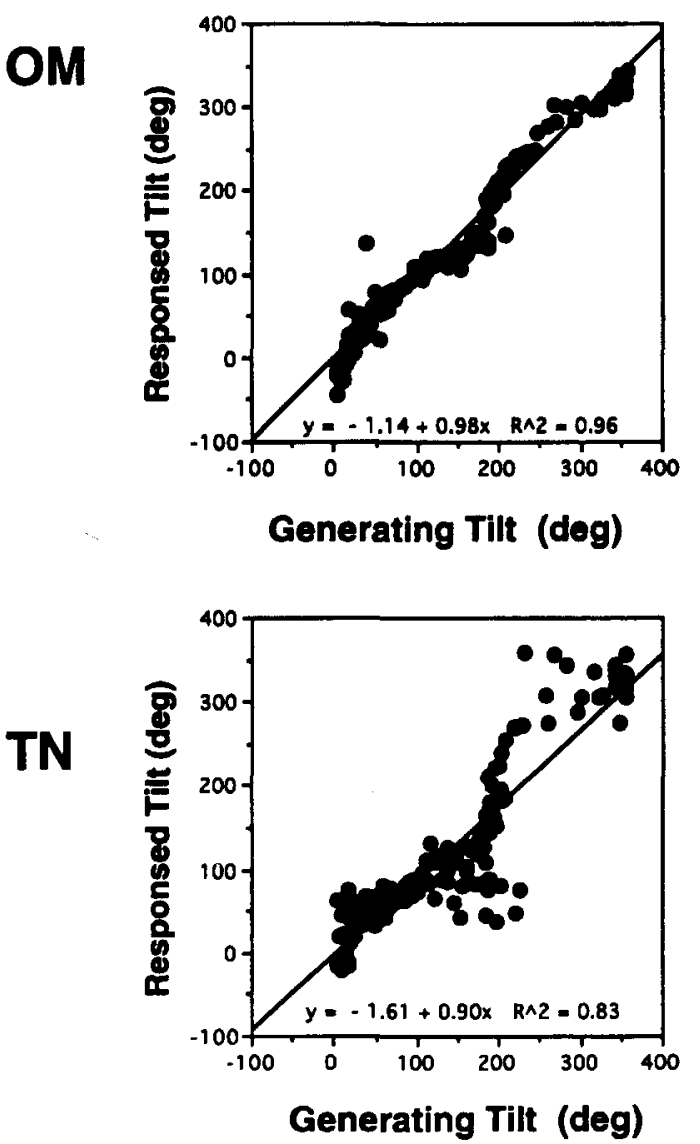

Gauge Figure
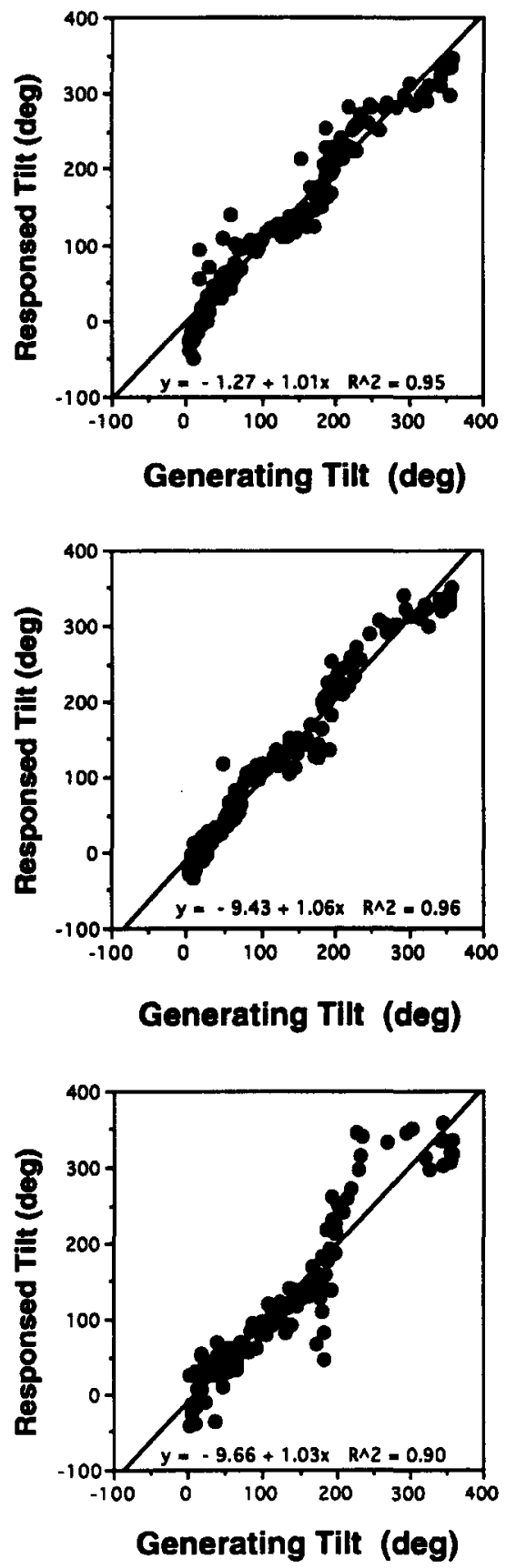

Figure 6B. Plots of tilt versus generating tilt from Experiment 1 along with linear regressions, for individual subjects (excluding Subject O.K.). Responses were not plotted if the associated slant response was less than $20^{\circ}$. 
of closest point being consistent with that of the best fitting surface.

Image construction from judgments of light-source direction and best fitting surface. From the data on best fitting surface, light-source direction, and closest point, it was apparent that 3 of the subjects were reporting an arrangement that was roughly consistent with the arrangement used to generate the stimulus. However, the same could not be said for Subject O.K., and the question arose as to whether the arrangement reported by Subject O.K. was a valid interpretation. To investigate whether Subject O.K.'s data was consistent with a lambertian interpretation, we used the data from the best fitting surface to obtain estimates of the surface normal, $\mathbf{N}$, at the 196 probe locations, then, along with the estimated light-source direction, $\mathbf{L}$, we calculated the lambertian image intensity, $\mathbf{L} \cdot \mathbf{N}$, at each probe point. These recovered values of $\mathbf{L} \cdot \mathbf{N}$ (the recovered image intensity) were then plotted versus the presented values of $\mathbf{L} \cdot \mathbf{N}$ (the presented image intensities) and linear regressions calculated.

Results showed that for Subjects O.M., T.N., and Y.W., $r^{2}=.94, .77$, and .94 , respectively, for the pointing sessions and $r^{2}=.90, .88$, and .75 , respectively, for the gauge sessions. In contrast, for Subject O.K., $r^{2}=.2$ and .14 for pointing and gauge, respectively. The linear regressions are shown in Figure 5 for Subjects O.K. and O.M. It can be seen that for Subject O.K. there was no linear relationship between the presented image intensity and the intensity obtained through a model of lambertian shading applied to the reported arrangement of surface and light-source direction. Thus, it seems unlikely that Subject O.K. was using a lambertian assumption to form her judgments. Though it is likely that the arrangement reported by Subject O.K. is compatible with a consistent set of assumptions for interpreting shape from shading, we did not examine the issue further.

Slant and tilt. The orientation judgments were evaluated by comparing the responded values with the simulated values of slant and tilt of the generating surface. Due to the obvious difference between the generating surface and what was reported, Subject O.K. was excluded from this and further analyses that compared the responses to the generating stimulus. For the remaining 3 subjects, the reported slants and tilts plotted versus their generating values are displayed in Figure 6 ( $A$ and $B$ ), along with the corresponding values of the linear regression. An ANOVA was performed on the slopes and $r^{2}$ values of the regression, using the method of response (gauge, pointing) and the data type (slant, tilt) as factors. There was no statistically significant effect of either of these factors on the slopes of the regression lines. For $r^{2}$, there was no effect of method of response, but there was a significant difference in $r^{2}$ between slant and tilt regressions $[F(1,2)=26.6$, $p<.05$ ].

The slant data showed considerable scatter of the responses, and at best, generating slant accounted for only $59 \%$ of the total variability. Subject Y.W.'s pointing responses showed a remarkable tendency to have large negative slant differences during the pointing session. Upon
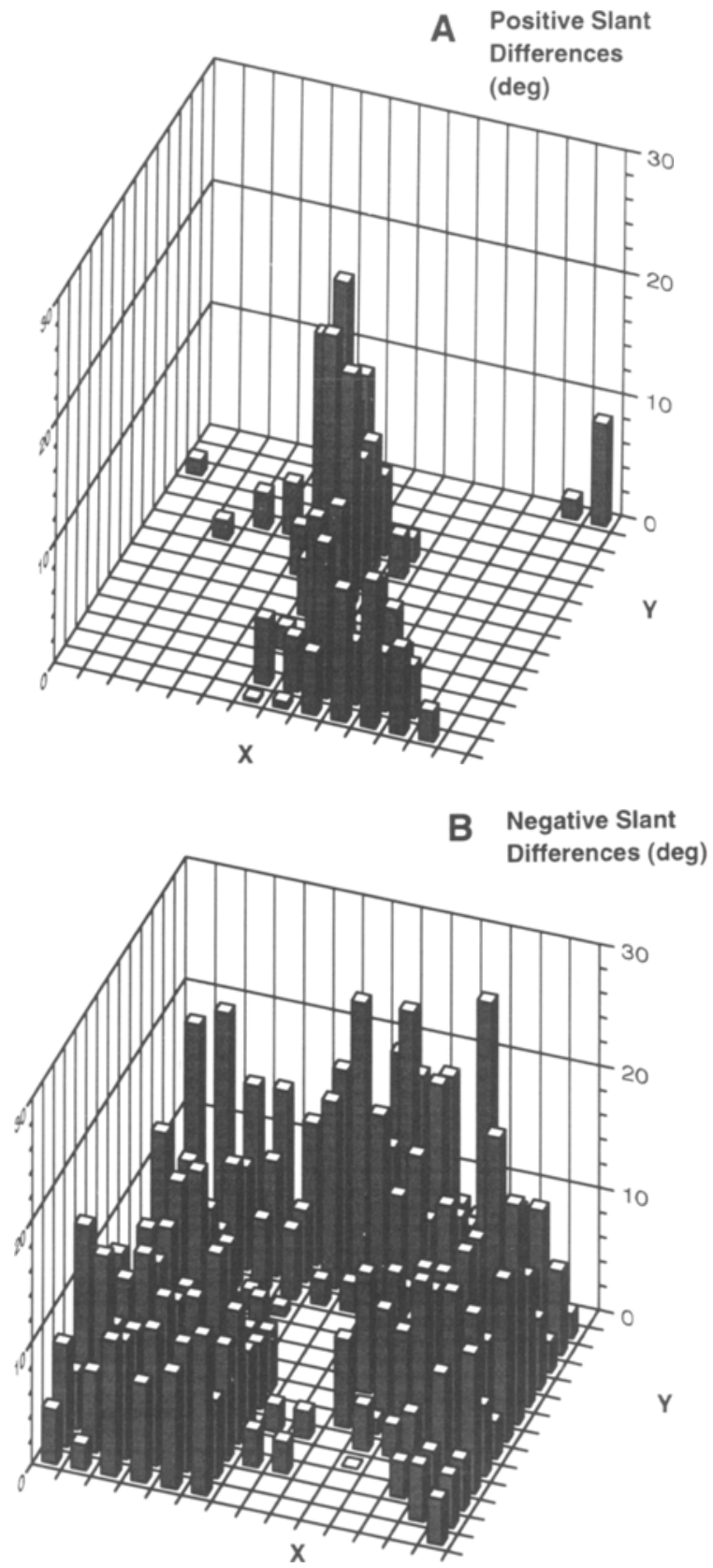

Figure 7. The spatial distribution of the average slant differences from Experiment 1 (excluding Subject O.K.). (A) The region of positive slant difference and $(B)$ the region of negative slant difference.

debriefing, this subject indicated that the top of the stimulus looked much flatter during the pointing session than during the gauge session, and examination of her data indicated that the large negative differences were due primarily to responses from the top rows of the stimulus. Thus, this substantial difference does not seem likely to have been caused solely by the method of response. Although the tilt regressions accounted for approximately $90 \%$ of the variability, there appeared to be a characteristic deviation of the tilt responses from a straight line, with tilt difference being 
a periodic function of generating tilt. The periodic nature of the difference suggests effects involving perceived symmetry, but this was not explored in detail.

Spatial distribution of slant differences. Although, on average, judgments of slant were less than the slant of the generating surface, there were a substantial number of responses where slant was greater. Since negative slant differences are what are generally reported ("regression to the picture plane"), we decided to examine the positive slant differences more closely. This was done by examining the locations in the image where slant difference was positive. The results are shown in Figure 7A, averaged across all subjects as well as response techniques, where it can be seen that the positive slant differences were localized to a diagonal region.

Negative slant differences, shown in Figure 7B, were greatest at the boundaries of the grid. In particular, the top of the grid showed a large amount of negative slant difference. This effect was consistent with comments of some subjects as well as many informal observers - that the stimulus lost its three-dimensional structure (became flat) as one moved to the boundaries of the image.

\section{Discussion}

The pre-/posttest results indicated that subjects' consistency on reporting surface orientation was within about $10^{\circ}$. Three of the 4 subjects reported a surface similar to that used to generate the shaded image, the other subject reported a surface that was different from the generating surface but nonetheless had measures of self-consistency similar to that reported by the other subjects. For the subject who did not report the generating surface, construction of a lambertian image from the reported light-source direction and best fitting surface indicated that the reported arrangement was incompatible with a lambertian interpretation. For the 3 subjects who reported a surface similar to the generating surface, examination of the slant and tilt regressions showed that the amount of variability accounted for by the regression was significantly different, with greater amounts of variability accounted for in the tilt regressions. Although slant differences were generally negative for these 3 subjects, there was a distinct region along the diagonal of the image where it was positive.

If we compare the results of the pointing technique with those of the gauge technique in terms of consistency of the responses and correlation with the generating surface, there was no remarkable difference between the two. The techniques of pointing and gauge provided results of similar consistency with both the pre-/posttest and best fitting surface. Correlations from the slant and tilt regressions were essentially identical between the two methods. Although failure to reject the null hypothesis does not assure that the methods yield identical results, the evidence from pre-/posttest, the internal consistency of the best fitting surface, and the slant and tilt regressions converge to suggest that a substantial difference does not exist.

For the 3 subjects who reported a surface similar to the generating surface, the results reproduced the general finding that reported slant was less than the slant of the gen-
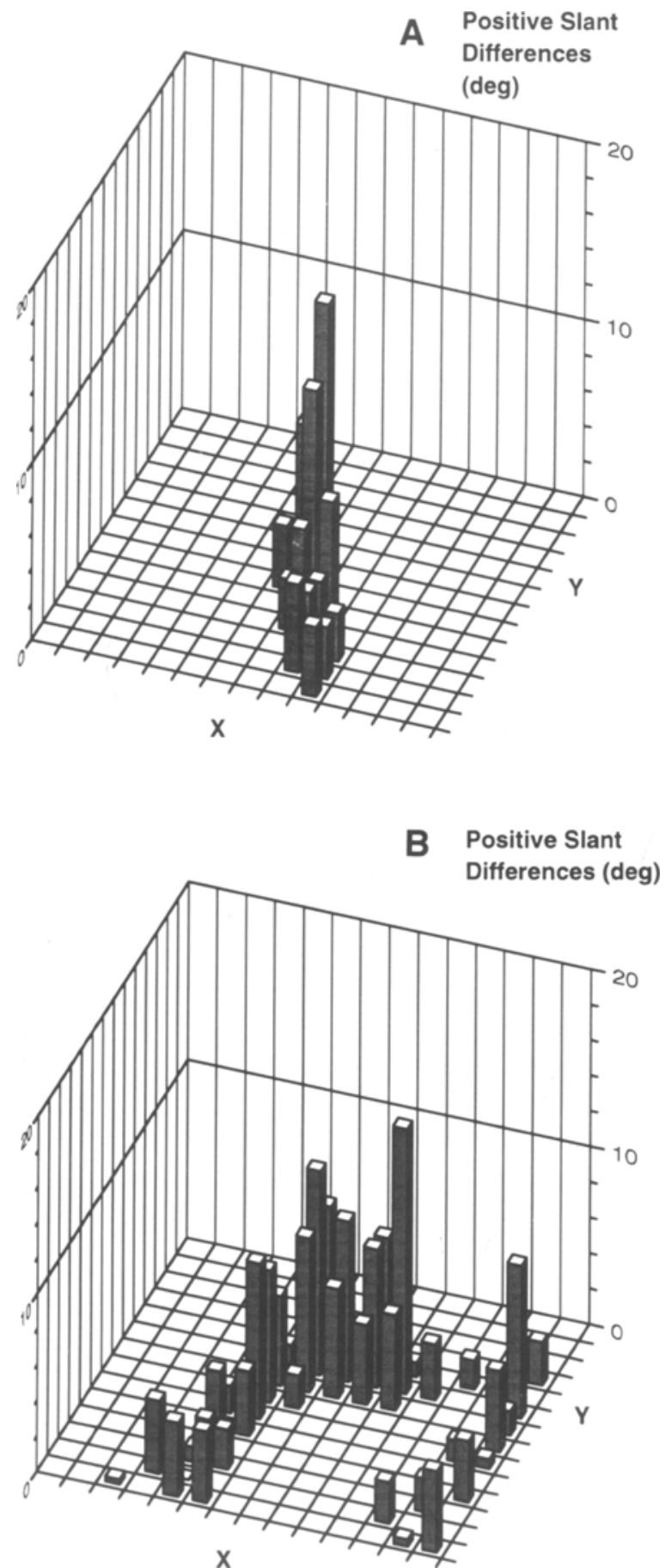

Figure 8. The spatial distribution of positive slant difference from Experiment 2: (A) Results for the orientation identical to that used in Experiment 1, and (B) results for the rotated orientation.

erating surface. However, counter to the general claim that slant difference is negative ("regression to the picture plane"), we found a distinct region that showed positive slant differences. Since its boundary, the zero slant difference contour, can be considered the locus of best performance at reproducing the generating surface, we decided 


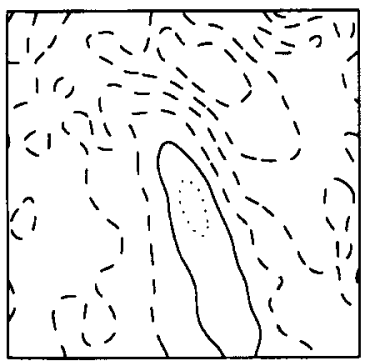

slant difference

A
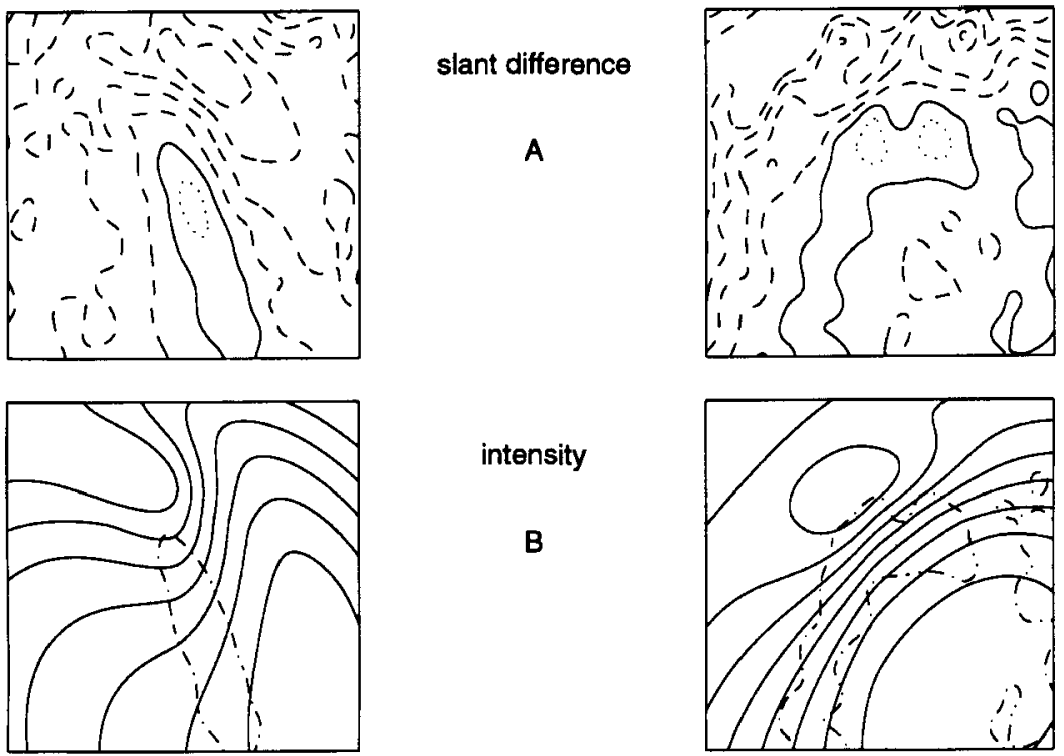

intensity
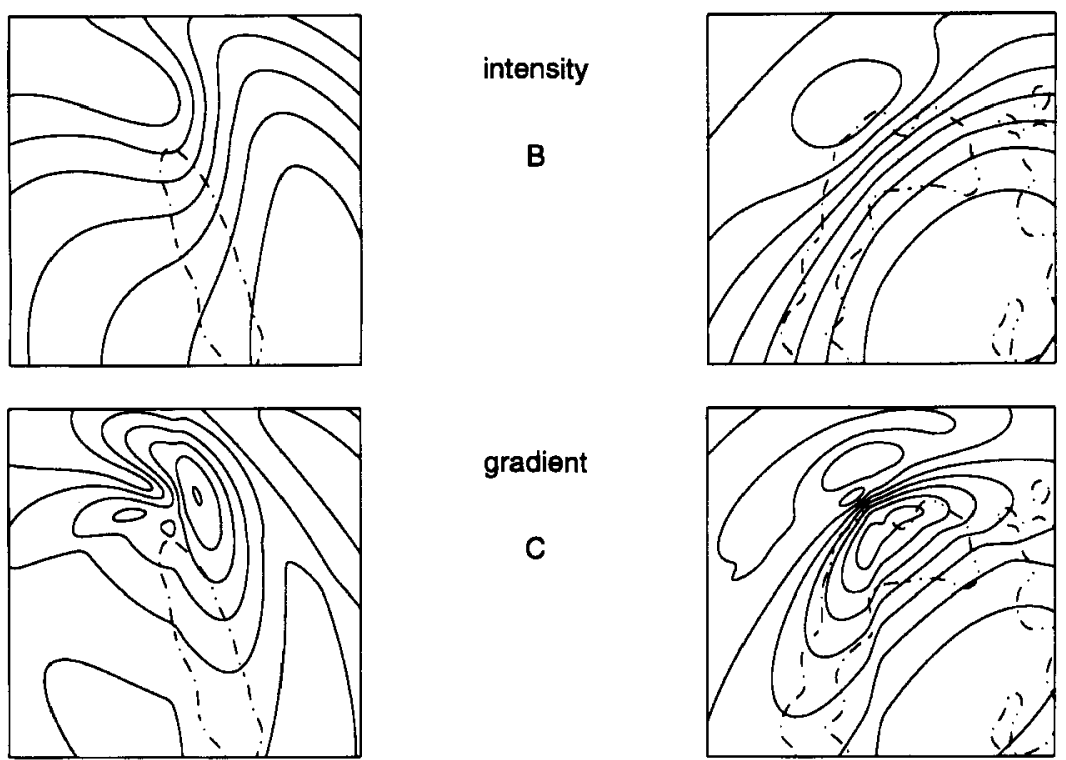

gradient
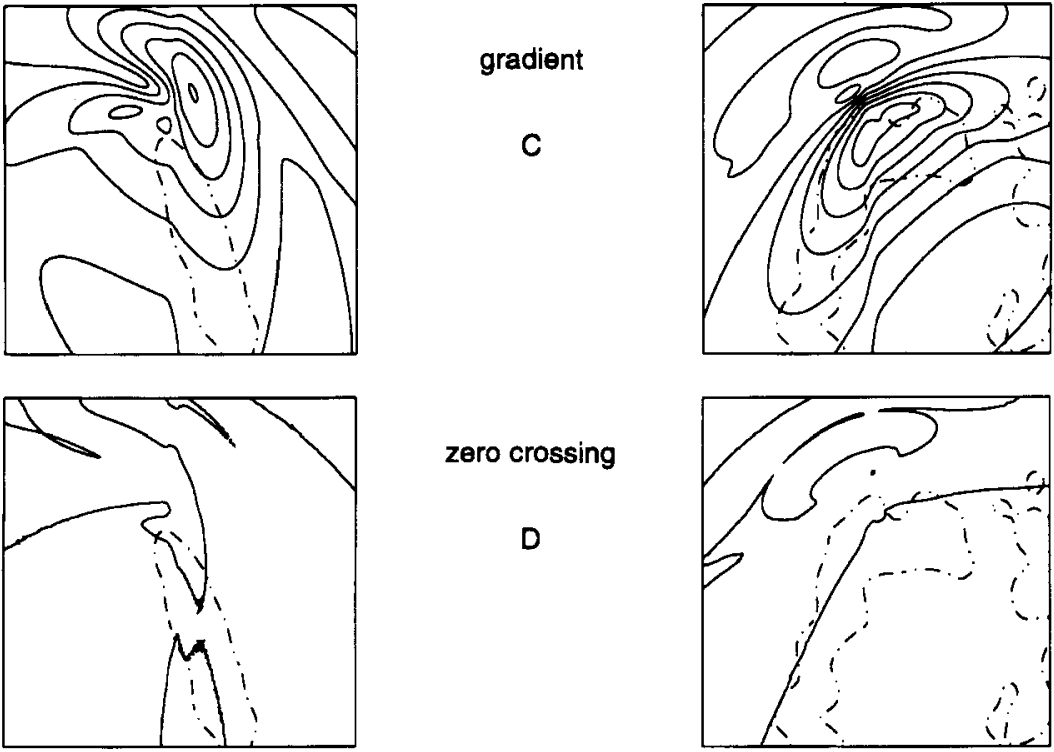

zero crossing
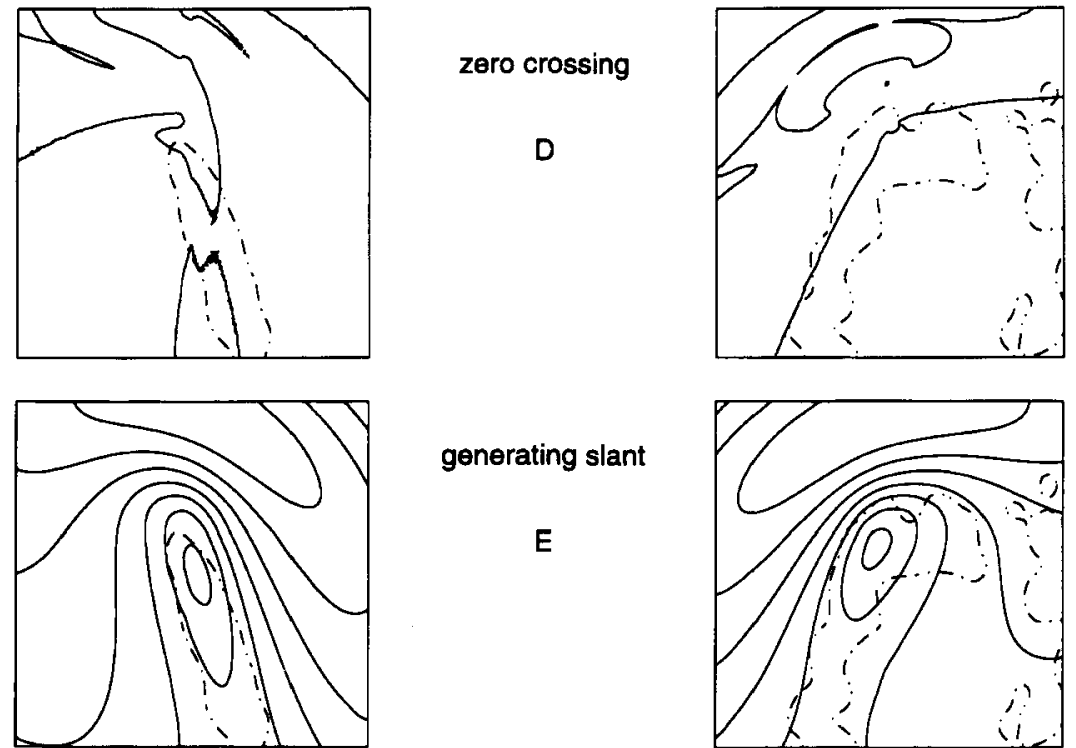

generating slant

E

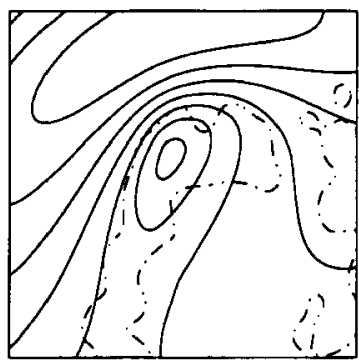

Figure 9. Results from Experiment 2 showing slant difference and its relation to image properties and the generating slant. The left column displays the results for the surface orientation identical to that in Experiment 1. The right column displays the results for the rotated orientation. (A) The contour map of slant difference, with the solid line indicating the line of zero slant difference. The dotted line indicates positive slant difference, and the dashed line indicates negative slant difference. The contour lines indicate values of slant at $10^{\circ}$ intervals. For the following panels, the contour of zero slant difference is indicated by a superimposed dot-dash line. (B) The contour map of the spatially filtered image intensity. $(C)$ The contour map of the gradient of the filtered image intensity. (D) The zero crossings of the second derivative of the filtered image intensity taken in the direction of gradient. (E) The contour map of generating slant. 


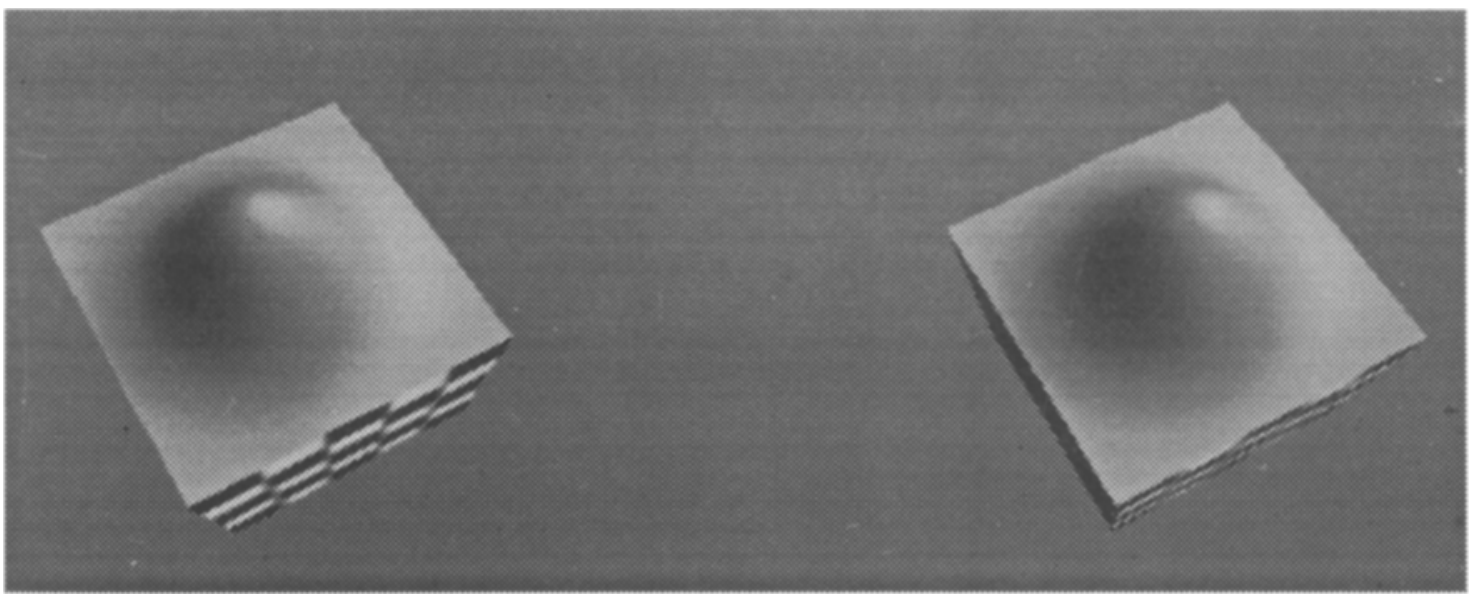

Figure 10. The Gaussian hill stimulus used in Experiment 3 with cues of shading, texture, perspective, and horizontal disparity.

to examine the phenomenon of positive slant differences more closely.

\section{EXPERIMENT 2}

The results of Experiment 1 showed a distinct contour of zero slant difference. On the image, this region corresponded roughly to a line connecting the brightest and darkest points. The purpose of Experiment 2 was to determine whether this line was a result of simple 2-D image properties such as intensity extrema or perhaps of a more subtle property of the image or underlying geometry. To this purpose, we explored reports of surface orientation for the same surface in two different orientations with the same light-source direction. This manipulation kept the luminance extremum at approximately the same positions in the image while rotating the surface. The hypothesis being that if the contour of zero-slant difference did not change, then it was likely due to some image-intensity artifact. If it did change, then how it changed would suggest what image properties contributed to the perception of shape from shading.

\section{Method}

Subjects. The subjects were the first author, Subject Y.W. from Experiment 1, and 2 additional persons who satisfied the same criteria as in Experiment 1.

Design. The single variable, which had two levels, was the orientation of the surface.

Stimuli and Apparatus. The shaded images were constructed in the same manner as in Experiment 1 and rendered the same surface in two different orientations. These orientations were: (1) the same as that used in Experiment 1, and (2) an orientation composed of a $90^{\circ}$ rotation of the surface (counterclockwise) about the viewing direction preceding the $22.5^{\circ}$ rotation about the horizontal. The lightsource direction of the generating configuration was the same as in Experiment 1 . The $14 \times 14$ grid of probe points was centered on the approximate center of the projection of the hill for each surface orientation. The subjects indicated local surface orientation with the pointing technique.

Procedure. The subjects were instructed on the pointing task and given several practice trials until they became confident with the task.
Next, each subject performed the pointing task once for each surface orientation, with the order of presentation of the surface orientations being counterbalanced. At the end of each session, the subjects gave judgments of light-source direction and closest point, just as in Experiment 1.

\section{Results and Discussion}

We first analyzed the data, as was done in Experiment 1, to check (1) whether the recovered surface conformed to a set of integrable surface patches, (2) correlation with the generating surface, and (3) whether judgments of lightsource direction and closest point were similar to the generating configuration. These results indicated that all subjects were reporting a surface similar to the generating one, and so it was decided to pool the data of individual subjects to examine for regions of positive-slant differences.

The regions of positive-slant differences for the two orientations are presented in Figure 8. Figure 8A shows the results from the surface in an orientation identical to that in Experiment 1, and Figure 8B shows the results of the rotated surface. It can be seen that the primary region of positive-slant difference did not stay in place, but that it rotated with the surface. This result suggests that slant estimation was not related to a simple property of the luminance field such as intensity extrema, and the following section outlines possible origins for the pattern of slant response.

The result that positive-slant differences followed surface orientation suggested that either a property of the generating surface or a property linked to the field of illumination could be responsible for the pattern of slant differences. For this reason, we looked at the relationship between slant differences and the distribution of slant on the generating surface, as well as the relationship between slant differences and the zero-, first-, and second-order properties of the illumination field. These results are presented in Figure 9, where one can inspect the contour maps of slant difference, generating slant, image intensity, image gradient, and the zeros of the second derivative taken in the direction of the image gradient.

Examination of the contour lines of generating slant and the line of zero slant difference reveals that the contour of 
Table 2

Regression Parameters of Reported Slant Versus Generating Slant From Experiment 3

\begin{tabular}{cccc}
\hline Subject & Slope & Intercept & $r^{2}$ \\
\hline F.P. & .49 & 13.19 & .62 \\
K.M. & .91 & 13.75 & .48 \\
M.M. & .79 & 1.56 & .72 \\
M.N. & .75 & 11.51 & .51 \\
Averages & .75 & 9.44 & .66 \\
\hline
\end{tabular}

zero-slant difference was very nearly, though not quite, a line of constant slant (Figure 9E). In addition, the generating slants less than this constant value were overestimated, while the generating slants greater than this value were underestimated. This indicates that slant judgments, rather than tending toward the slant of the picture plane, tended toward an intermediate slant value. Although this result is consistent with such a statistical explanation as regression to the mean, it does not preclude the possibility that the contour of zero slant difference was, instead, obtained through a mechanism of the recovery of shape from shading, which provided exceptional performance along this particular contour.

Inspection of the image intensity and its first and second derivatives indicates that the region of zero slant difference was approximately aligned with the zeros of the second derivative in the direction of the gradient. Of course, since the zeros of the second derivative are related to the extrema of the first derivative, one can also describe the zero slant difference line as approximately descending the peak of the gradient in the direction of steepest descent. In this respect, it is interesting to note that there were zero crossings of the second derivative that were not related to the region of zero slant difference.

\section{EXPERIMENT 3}

The results of the first two experiments showed that there were distinct regions where, relative to the generating surface, judgments of surface orientation had positive and negative slant differences, and that the boundary of these regions was approximately a line of constant slant that was in proximity to the zero crossings of the second directional derivative. However, one aspect of the Gaussian hill stimuli used in these experiments was that, as intensity became uniform at distances far from the top of the hill, there was a tendency of subjects to report surface orientation as frontoparallel rather than slanted, and this effect could have propagated inwards. Since the pattern of zero slant differences might have been caused by this flattening effect, we decided to place a Gaussian hill surface within a context where additional information existed to disambiguate the global orientation of the boundaries. The rationale was that if the pattern of slant response disappeared when the global orientation was made more apparent, then it was probably due to an artifact of the flattening seen in the boundaryless displays.

\section{Method}

Subjects. There were 4 subjects, the first author and three persons who were naive as to the purpose of the experiment. The naive subjects satisfied the criteria that were used in Experiment 1.

Design. For a single surface, the subjects judged local surface orientation three times to each of 149 locations. These probe locations covered the extent of the surface.

Stimuli and Apparatus. The stimulus simulated a lambertian shaded hill atop a box with textured sides (Figure 10). The box was rotated $22.5^{\circ}$ about the horizontal so that the simulated depth of the top corner was farther away than the bottom corner. The precise form of the hill was a symmetric Gaussian with amplitude 2.5. The shape was presented using horizontal disparity and perspective cues appropriate for a viewing distance of $60 \mathrm{~cm}$. Stereo was obtained by using $120-\mathrm{Hz}$ sequential presentation of left and right images by liquid crystal shutter glasses (CrystalEyes) synchronized to the display monitor (this mode of presentation yielded a screen resolution of $491 \times 1280$ ), and the interocular separation was adjusted to the measured value for each subject. The generating light source was at infinity at an orientation of $(0.49,0.29,0.82)$. The pattern of shading was calculated by the SGI using a Gourad algorithm (Foley, van Dam, Feiner, \& Hughes, 1990) to interpolate gray levels between the 10,000 vertices used to create the hill.

Local orientation was probed at 149 locations which were arranged in a hexagonal array that covered the surface of the hill. From the
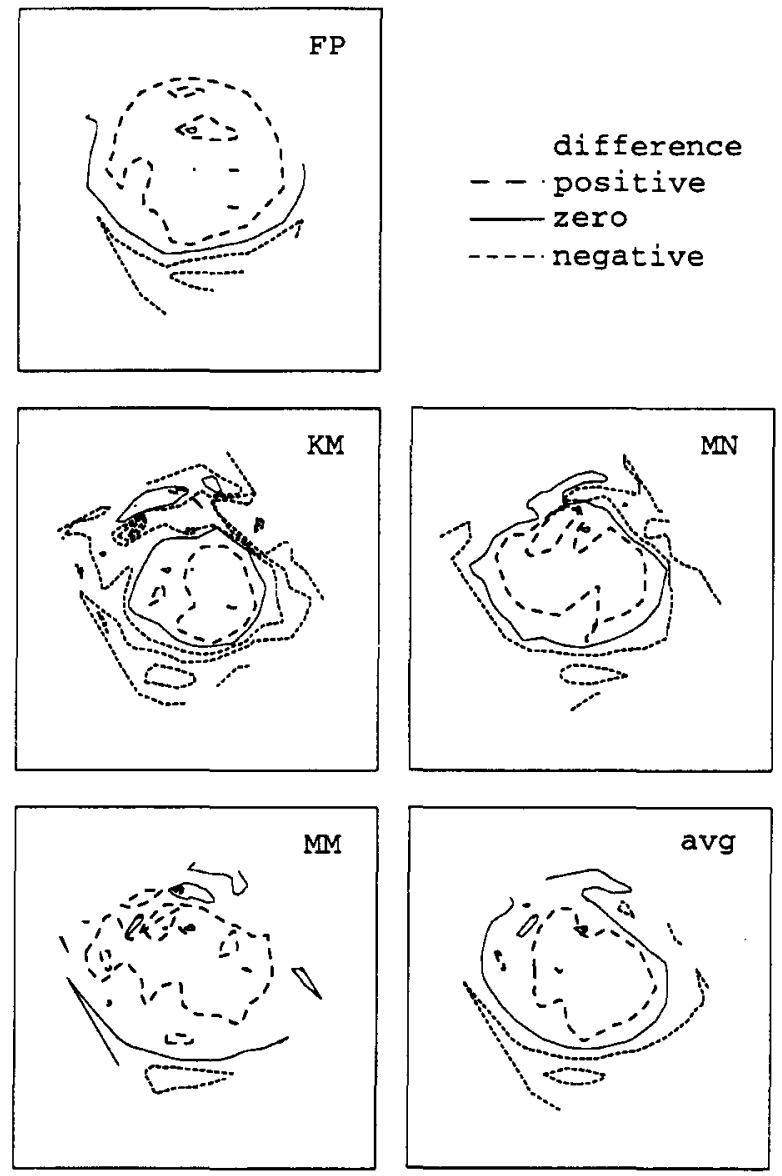

Figure 11. Slant difference results from Experiment 3 for individual subjects and their overall average. The slant contours are spaced at $10^{\circ}$ intervals. 


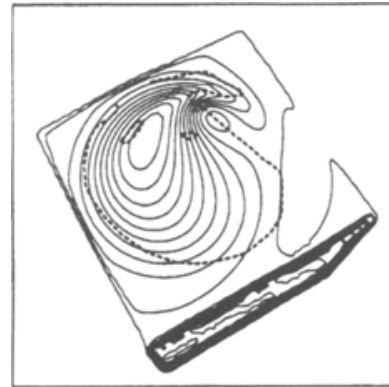

intensity

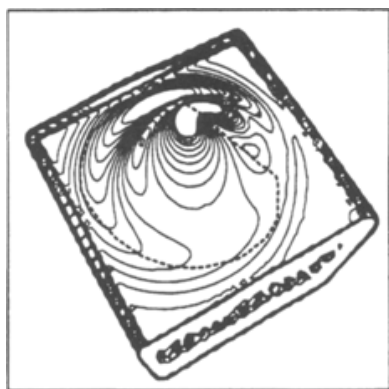

gradient
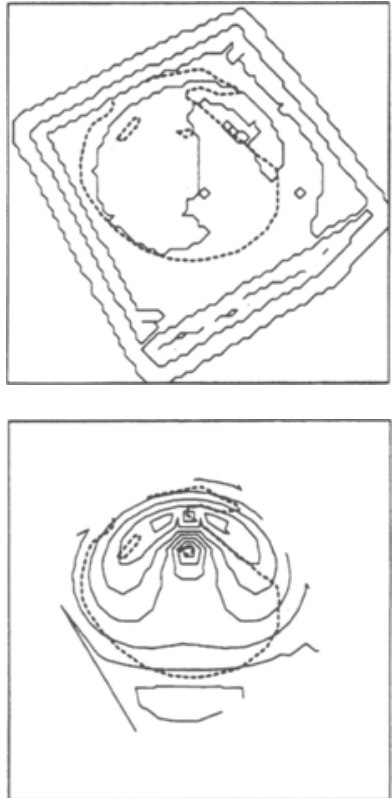

zero crossing

generating slant

Figure 12. Zero slant difference of the average response from Experiment 3 superimposed with the intensity, gradient, and zero crossing of the illumination field, as well as the generating slant.

viewing distance of $60 \mathrm{~cm}$, the hill subtended a visual arc $17^{\circ}$ high and $17^{\circ}$ wide. The probe point was visible to only the left eye, and the generating values of orientation were taken from the view corresponding to the image obtained if the eye separation was set to zero. The orientation judgments were obtained by using pointing responses.

Procedure. Each subject participated in a preliminary training session followed by three sessions of data collection. The training session consisted of instructing the subjects how to make pointing responses to the perceived surface orientation, and practice trials were performed until the subjects were comfortable with the response method. Each session of data collection consisted of the subjects' providing responses of surface orientation to each of the 149 loca- tions, presented in a random order. The sessions were self-paced, and between sessions there was a minimum rest of $30 \mathrm{~min}$.

\section{Results and Discussion}

For each of the 149 probe positions, data were averaged across individual sessions to obtain an average response for each subject and across subjects to obtain an overall average. For each individual subject and the overall average, a linear regression was calculated from the responded slants and tilts versus the slants and tilts of the generating stimulus. The resulting values are given in Table 2 .

The slant differences at the 149 probe locations were used to estimate the contours of constant slant difference, in particular the contour of zero slant difference. The results for individual subjects and overall are shown in Figure 11. While it can be seen that individual subjects had distinctive patterns of slant difference, there were common areas across the lower half and in the upper left corner that contributed to the

A
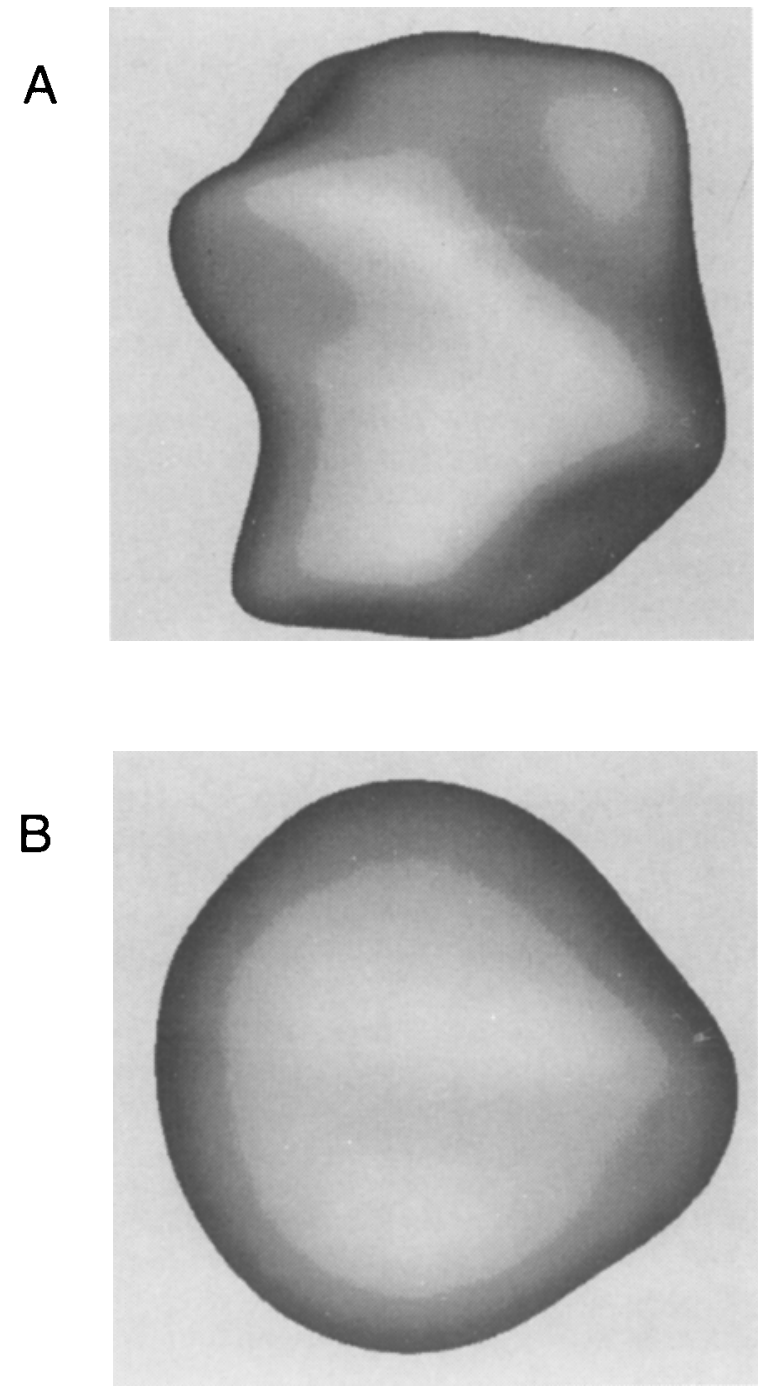

Figure 13. The stimulus blobs (A and B) used in Experiment 4. 
Table 3

Regression Parameters of Reported Slant and Tilt Versus Generating Slant and Tilt From Experiment 4

\begin{tabular}{|c|c|c|c|c|c|c|c|}
\hline \multirow[b]{2}{*}{ Subject } & \multirow{2}{*}{$\begin{array}{c}\text { Response } \\
\text { Component }\end{array}$} & \multicolumn{3}{|c|}{ Blob A } & \multicolumn{3}{|c|}{ Blob B } \\
\hline & & Slope & Intercept & $r^{2}$ & Slope & Intercept & $r^{2}$ \\
\hline \multirow[t]{2}{*}{ F.P. } & Slant & .92 & 13.06 & .48 & .77 & 10.92 & .52 \\
\hline & Tilt & .80 & 12.24 & .71 & .96 & 5.83 & .86 \\
\hline \multirow{2}{*}{ K.M. } & Slant & .32 & 14.63 & .33 & .14 & 28.81 & .04 \\
\hline & Tilt & 1.04 & 9.75 & .65 & 1.06 & 3.11 & .82 \\
\hline \multirow{2}{*}{ M.M. } & Slant & .11 & 19.07 & .05 & .20 & 9.92 & .11 \\
\hline & Tilt & .87 & 17.50 & .52 & .70 & 28.10 & .67 \\
\hline \multirow[t]{2}{*}{ M.N. } & Slant & .49 & 9.98 & .68 & .42 & 15.40 & .43 \\
\hline & Tilt & .94 & -13.66 & .66 & .91 & -4.66 & .83 \\
\hline \multirow[t]{2}{*}{ Averages } & Slant & .20 & 20.2 & .20 & .41 & 10.84 & .47 \\
\hline & Tilt & .86 & 7.81 & .53 & .81 & 19.02 & .70 \\
\hline
\end{tabular}

pattern of zero slant difference found in the overall average. This pattern of slant difference was similar to that reported in the two previous experiments in that there were distinct regions of positive and negative differences. However, one notable difference was the positive slant differences at the bottom boundary of the box. This finding suggests that the additional visual cues were effective in removing the frontoparallel tendency found in the previous experiments.

The relationship between zero slant difference and properties of the illumination field and generating slant can be examined in Figure 12. It can be seen that, as before, this contour of zero slant difference shared large regions of adjacency with the zero crossings of the second derivative. An important observation is that although the generating slant was symmetric about the midline of the stimulus, the slant difference was not. This result indicates that the contour of zero slant difference need not be entirely dependent on the pattern of the generating slant.

The results of Experiment 3 showed a similar pattern of zero slant difference, as in Experiments 1 and 2. This was despite the fact that the large amounts of negative slant differences at the boundaries were removed by supplementing the shading information with additional visual cues to the global orientation. Thus, from the results of Experiment 3 , it seems unlikely that the pattern of zero slant difference found in these previous experiments could be accounted for simply by weak cues to orientation at the boundaries of the displays.

\section{EXPERIMENT 4}

Although the results of Experiment 3 showed a contour of zero slant difference that was similar in nature to that found in Experiments 1 and 2, there are still limitations to interpreting this as a general result of shape from shading. For example, the surface was a Gaussian hill, similar to that used in Experiments 1 and 2, and the contours defining the boundaries of the shaded image were sharp edges rather than smooth self-occluding boundaries. In the fourth experiment, we examined the ability to report local orientation when given monocular views of lambertian-shaded, irregularly shaped blobs that contained smooth self-occluding boundaries.

\section{Method}

Subjects. The same 4 subjects used in Experiment 3 participated in Experiment 4.

Design. For two blob-shaped surfaces, subjects judged local surface orientation three times for each member of a set of probe locations covering the visible extent of the surface.

Stimuli and Apparatus. The two generating stimuli were lambertian shaded, arbitrarily shaped blobs with smooth self-occluding contours (Figure 13). The blobs were created by using a b-spline surface to generate a triangular mesh of vertices that was rendered by the graphics system. For both blobs, the generating light source was in a direction coincident with the viewing direction and located at infinity. As with the stimuli in Experiment 3, the pattern of shading was calculated by the SGI, using a Gourad algorithm (Foley et al., 1990) to interpolate gray levels between the 7,290 vertices used to create blobs A and B. The combination of surface and light source was arranged so that no attached shadows would appear on the surface.

Local orientation was probed at 115 locations on blob $A$ and 112 locations on blob B. The probe points were arranged in a rectangular array that covered the visible front surface of the blobs. The viewing distance and apparatus used to display and control visibility of the stimuli was identical to that used in Experiments 1 and 2. These stimuli subtended visual arcs $11^{\circ} \mathrm{high}$ and $10^{\circ}$ wide for blob $\mathrm{A}$ and $11^{\circ} \mathrm{high}$ and $11^{\circ}$ wide for blob $\mathrm{B}$. The subjects gave their orientation judgments using the pointing technique.

Procedure. Each subject participated in a preliminary training session followed by six sessions of data collection. In the training session, the subjects were instructed on how to provide pointing responses to the perceived surface orientation, and practice trials were performed until the subjects were comfortable with using the response method. Each session of data collection consisted of the subjects' providing responses of surface orientation to every probe location of either blob A or blob $\mathrm{B}$. The sessions alternated between blob $A$ and blob $B$, with 2 subjects beginning with blob $A$ and 2 subjects beginning with blob $B$. The sessions were self-paced and spread over at least 2 days. Between sessions, there was a minimum rest of $30 \mathrm{~min}$.

\section{Results and Discussion}

For each of the probe positions of blobs A and B, data were averaged across the three repetitions to obtain an average response for each subject and across subjects to obtain an overall average. For each individual subject and the overall average, a linear regression was calculated from the responded slants and tilts versus the slants and tilts of the generating stimulus. The resulting values are shown in Table 3. An ANOVA was performed on the slopes and $r^{2}$ values of the regressions using blob (blob A, blob B) and 
the data type (slant, tilt) as factors. The results showed a significant difference in $r^{2}$ between slant and tilt regressions $[F(1,3)=19.6, p<.05]$.

For both blob $\mathrm{A}$ and blob $\mathrm{B}$, the slant differences at the probe locations were used to estimate the contours of constant slant difference, including the contour of zero slant difference. The results for individual subjects and their overall average are shown in Figure 14. As can be seen, although differences existed, individual subjects had similar patterns of slant difference for both surfaces. Overall, the pattern of slant estimation was similar to that obtained with the boundaryless Gaussian hills - an internal region of positive slant differences with slant differences becoming increasingly negative as one moved outward on the surface.

The relationship between zero slant difference and properties of the illumination field and generating slant can be examined in Figure 15. As in the previous experiments, it can be seen that the contour of zero slant difference shared large regions of adjacency with the zero crossings of the second derivative. However, it is worth noting that adjacent to the profile of the object was a zero crossing that did not seem to be associated with the contour of zero slant difference. One aspect that can be seen in Figure 15D is that the line of zero slant difference was also almost adjacent to a line of constant slant. In addition, since the viewing direction was coincident with the light-source direction, this contour was also identical to a line of constant brightness on the image.

The results of Experiment 4 showed that there was no change in the pattern of subject performance with the introduction of smooth self-occluding contours to constrain the shaded image. Moreover, it seemed that the self-occluding contour itself was not a compelling source of information for local orientation. For example, the region adjacent to the occluding contour for blob A yielded negative slant differences of approximately $30^{\circ}$.

\section{GENERAL DISCUSSION}

Experiment 1 investigated local orientation judgments from the shaded image of a boundaryless Gaussian hill. The results showed substantial between-subject differences, with 1 subject reporting an internally consistent surface that was quite different from the surface used to generate the image. For the subjects reporting a surface similar to that of the generating surface, it was found that there were distinct regions of positive and negative slant differ-
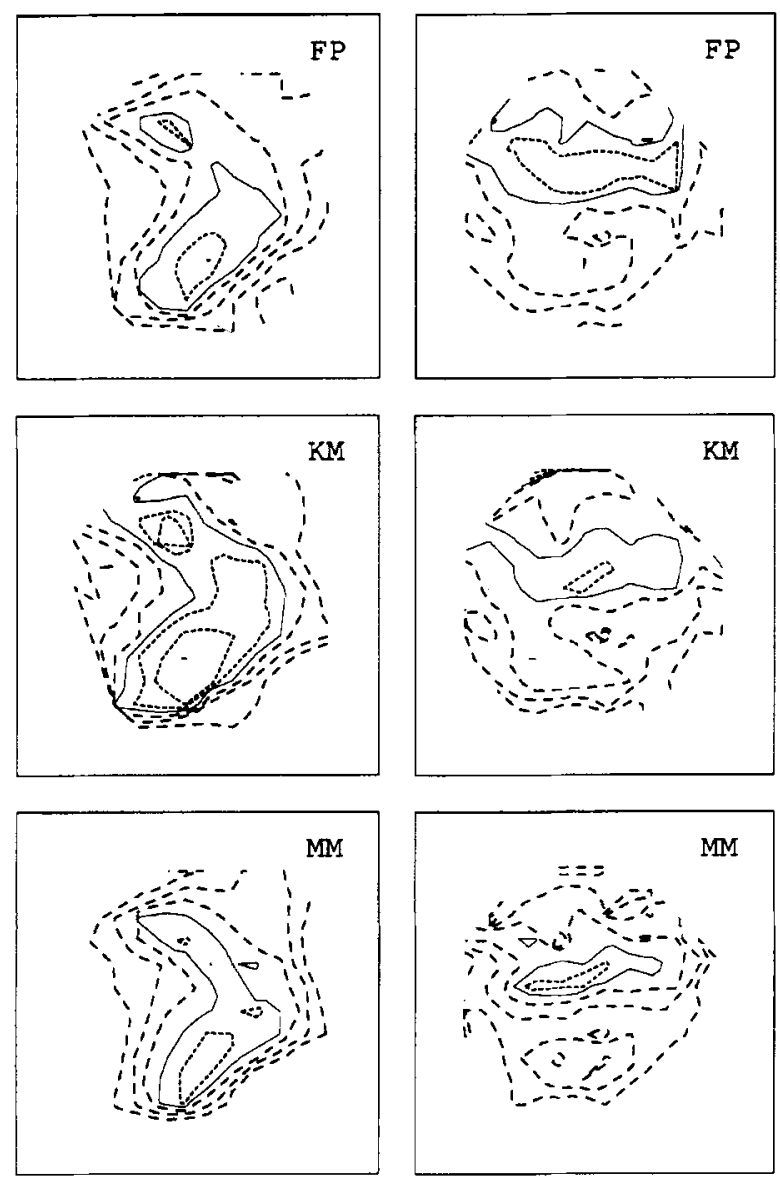
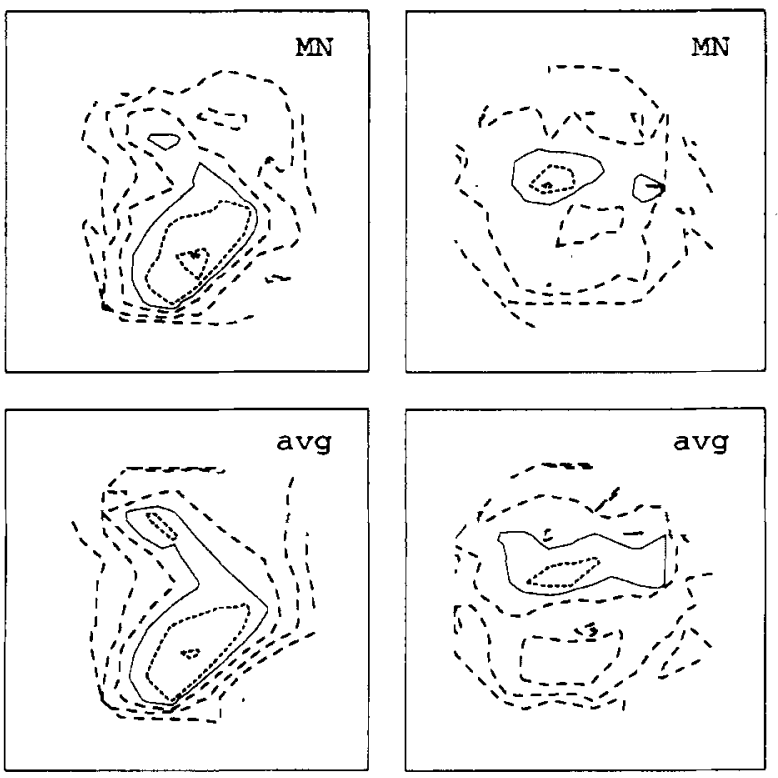

Figure 14. Slant difference results from Experiment 4 for individual subjects and their overall average. For each pair, the left panel corresponds to blob $A$, the right to blob $B$. The slant contours are spaced at $10^{\circ}$ intervals. 

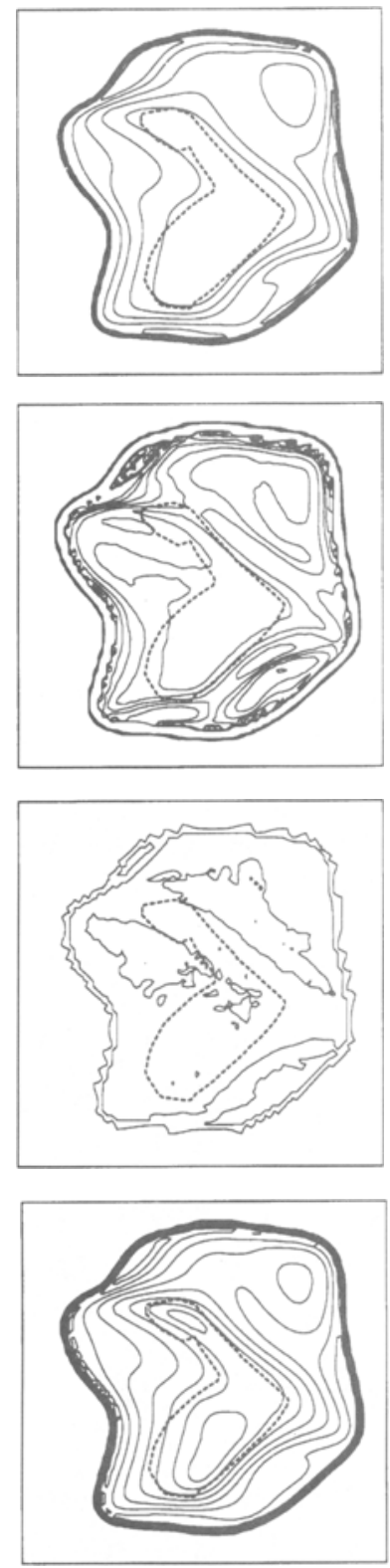

intensity

A

gradient

B

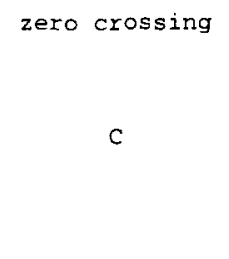

generating slant

D
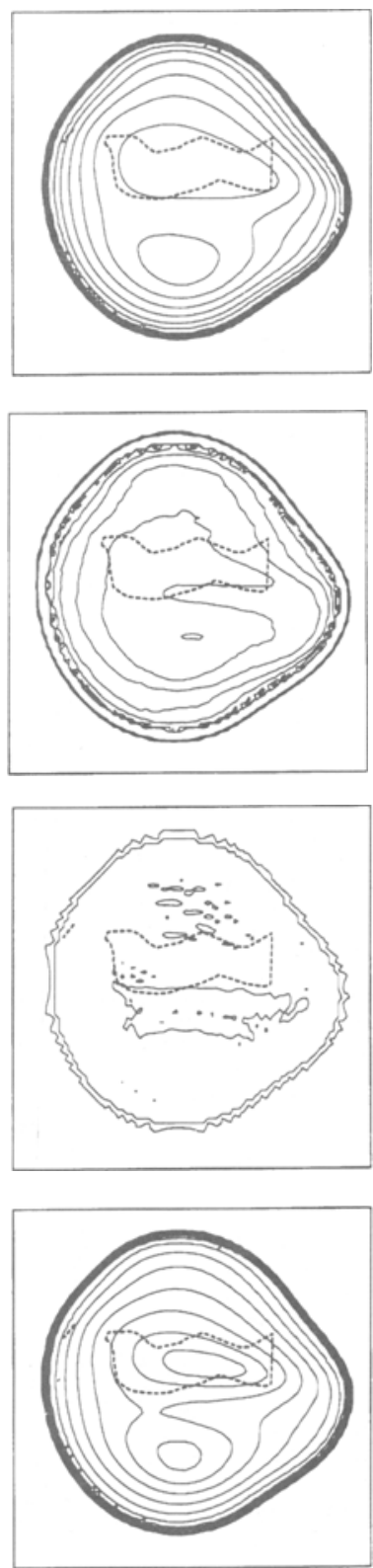

Figure 15. Zero slant difference of the average response from blobs $A$ (left column) and B (right column) of Experiment 4 superimposed with the intensity, gradient, zero crossing of the illumination field, and the generating slant.

ences between responded and generating slants. Since the boundary of these regions provides a line of best performance in reporting the generating surface and the common report in shape from shading is for negative slant differences ("regression to the picture plane"), we explored this phenomenon in greater detail. Experiments 2-4 explored whether the contour of zero slant difference found in Experiment 1 was caused by luminance artifact (Experiment 2), perceived flatness at the image extremes (Experiment 3 ), or the lack of self-occluding contours (Experiment 4). All three experiments showed patterns of slant response that had contours of zero slant difference sepa- rating distinct regions of positive and negative slant differences.

The results of Experiment 1 showed that, given images with orientation specified only by lambertian shading, subjects could reproduce a fixed orientation with a variability of approximately $10^{\circ}$, and that over the 196 probed locations linear regressions of responded tilt and slant versus generating tilt and slant accounted for as much as $96 \%$ of the variance in tilt and $59 \%$ of the variance in slant. While this precision appears to be below that reported by Pollick et al. (1994) for a real object under natural viewing conditions, it is slightly better than they obtained for pointing to 
the axis of rotation. There was no statistically significant difference between the pointing and gauge techniques used to obtain the orientation judgments. This was a somewhat unexpected result given the reports indicating that errors in pointing could be due to errors in performing sensorimotor transformations rather than in the perception of orientation (Flanders \& Soechting, 1994; Soechting \& Flanders, 1993). However, factors such as the unlimited time given for a response and the visual similarity between the gauge figure and the digitizing wand might have worked against detecting a difference.

In Experiment 1, comparisons of the best fitting surface (Koenderink et al., 1992) as well as subjects' reports of closest point and light-source direction revealed that 3 of the 4 subjects reported a scene similar to what was used to generate the image. The remaining subject reported a scene quite distinct from that used to generate the image. Analysis of whether this recovered surface and light-source direction were compatible with a lambertian interpretation indicated that they were not. This finding of between-subject differences is reminiscent of reports by Koenderink et al. (1992) for local shape recovery from pictures, except that their results suggested that the difference between individual subjects lay in different depth-scaling values, while the current results suggest that the between-subject differences are more extensive.

When subjects reported a surface similar to the generating surface, it was found that distinct regions of positive and negative slant differences occurred. The purpose of Experiments 2-4 was to explore possible explanations for this behavior. The results of the three experiments suggest that behavior was not caused by an artifact of illumination (Experiment 2) or perceived global orientation (Experiment 3 ), and similar results were obtained even with the introduction of self-occluding contours (Experiment 4).

The lack of a strong influence of the occluding contour is interesting, inasmuch as it is known that for a smooth surface the slant must be perpendicular to the viewing direction at the occluding contour and that one might thus have expected the contour of zero slant difference to be in direct proximity to the profile of the object. Although this seeming lack of an effect at the profile might not be entirely unexpected, given certain theoretical formulations of the shape-from-shading problem (Oliensis, 1991a), it is surprising in view of previous psychophysical demonstrations. Results of Ramachandran (1988) and Todd and Reichel (1989) have shown that the profile can exert a powerful influence on the percept of a shading pattern. In addition, the somewhat related results of Mamassian et al. (in press) have shown that categorical perception of local structure improves in the vicinity of the profile.

The results from Experiments $2-4$ showed that the line of zero slant difference was most closely related to two other contours. These were the contours of constant slant of the generating surface and the contours of the zero crossing of the directional derivative. The relationship between the zero-slant-difference contour and contours of constant slant indicated that subjects' behavior in reporting perceived slant was better characterized by regression to a constant value of slant rather than by regression to the picture plane. The relationship between zero slant difference and the zero crossings of the second derivative has possible significance to a computational analysis of shape from shading. Yuille (1989) has shown that the zero crossing of the second directional derivative is likely to occur near extrema on the surface, and thus it is possible that such knowledge was being used to constrain the interpretation of slant along this region. An additional factor to consider in this respect is the suggestion by Erens et al. (1993b) that recovery of shape from shading will be facilitated in regions where the magnitude of the gradient is large.

Although possible explanations for subjects' responses can be found in both the contours of constant slant and the contours of the zero crossings, it is necessary to point out that the data show inconsistencies in both relationships as complete descriptions of the results. For example, there were zero-crossing contours that were not associated with contours of zero slant difference (e.g., the zero crossing directly interior to the profile in Experiment 4) as well as the contours of zero slant difference that were not associated with contours of constant slant (e.g., the contour of zero slant difference found in Experiment 3). Moreover, the zero crossings shared large regions of adjacency with lines of constant slant, and this natural confounding makes it difficult to separate the possible explanations. However, one consistent interpretation of the present series of experiments is that performance at reproducing the generating surface is not uniform across a shaded surface. Instead, it seems to be influenced by potentially informative regions of the illumination field which are associated with zero crossings of the second directional derivative.

\section{REFERENCES}

Bülthoff, H. H., \& MALlot, H. A. (1988). Integration of depth modules: Stereo and shading. Journal of the Optical Society of America, 5, 1749-1758.

ERENS, R. G. F. (1993). Visual perception of shape from shading. The Hague: CIP-DATA Koninklijke Bibliotheek. (Doctoral dissertation, University of Utrecht)

Erens, R. G. F., Kappers, A. M. L., \& Koenderink, J. J. (1993a). Estimating local shape from shading in the presence of global shading. Perception \& Psychophysics, 54, 334-342.

Erens, R. G. F., Kappers, A. M. L., \& Koenderink, J. J. (1993b). Estimating the gradient direction of a luminance ramp. Vision Research, 33, 1639-1643.

Erens, R. G. F., Kappers, A. M. L., \& Koenderink, J. J. (1993c). Perception of local shape from shading. Perception \& Psychophysics, 54, 145-157.

FERRARO, M. (1994). Local geometry of surfaces from shading analysis. Journal of the Optical Society of America A, 11, 1575-1579.

Flanders, M., \& Soechting, J. F. (1995). Frames of reference for hand orientation. Journal of Cognitive Neuroscience, 7, 182-195.

Foley, J. D., van Dam, A., Feiner, S. K., \& Hughes, J. F. (1990). Computer graphics, principles and practice (2nd ed.). Reading, MA: Addison-Wesley.

Freeman, W. T. (1994). The generic viewpoint assumption in a framework for visual perception. Nature, 368, 542-545.

Hayakawa, H., Nishida, S., Wada, Y., \& Kawato, M. (1994). A computational model for shape estimation by integration of shading and edge information. Neural Networks, 7, 1193-1209. 
HoRN, B. K. P. (1987). Closed-form solution of absolute orientation using unit quarternions. Journal of the Optical Society of America A, $4,629-642$.

HoRN, B. K. P. (1990). Height and gradient from shading. International Journal of Computer Vision, 5, 37-75.

IKEUCHI, K., \& HORN, B. K. P. (1981). Numerical shape from shading and occluding boundaries. Artificial Intelligence, 17, 141-184.

KaWato, M., Hayakawa, H., \& INUi, T. (1993). A forward-inverse optics model of reciprocal connections between visual cortical areas. Network, 4, 415-422.

KoenderinK, J. J., \& VAN DoORN, A. J. (1980). Photometric invariants related to solid shape. Optica Acta, 27, 981-996.

Koenderink, J. J., \& VAN Doorn, A. J. (1993). Illuminance critical points on generic smooth surfaces. Journal of the Optical Society of America A, 10, 844-854.

Koenderink, J. J., van Doorn, A. J., \& Kappers, A. M. L. (1992). Surface perception in pictures. Perception \& Psychophysics, 52, 487-496.

Mamassian, P., \& Kersten, D. (in press). Illumination, shading and the perception of local orientation. Vision Research.

Mamassian, P., Kersten, D., \& Knill, D. C. (in press). Categorical local shape perception. Perception.

Mingolla, E., \& TODD, J. T. (1986). Perception of solid shape from shading. Biological Cybernetics, 53, 137-151.

OliENSIS, J. (1991a). Shape from shading as a partially well-constrained problem. CVGIP: Image Understanding, 54, 163-183.

OliENSIS, J. (1991b). Uniqueness in shape from shading. International Journal of Computer Vision, 6, 75-104.

Pentland, A. P. (1982). Finding the illumination direction. Journal of the Optical Society of America, 72, 448-455.

Pentland, A. P. (1984). Local shading analysis. IEEE Transactions on Pattern Analysis \& Machine Intelligence, 6, 170-187.

Pollick, F. E., Nishida, S., KoIKe, Y., \& KaWato, M. (1994). Perceived motion in structure from motion: Pointing responses to the axis of rotation. Perception \& Psychophysics, 56, 91-109.

Ramachandran, V. S. (1988). Perceiving shape from shading. Scientific American, 259, 58-65.

SAXBERG, B.V. H. (1989). A modern differential geometric approach to shape from shading (Report No. TR-1117). Cambridge, MA: M.I.T. Artificial Intelligence Laboratory.

Soechting, J. F., \& Flanders, M. (1993). Parallel, interdependent channels for location and orientation in sensorimotor transformations for reaching and grasping. Journal of Neurophysiology, 70, 1137-1150.

Stevens, K. A. (1983a). Slant-tilt: The visual encoding of surface orientation. Biological Cybernetics, 46, 183-195.

Stevens, K. A. (1983b). Surface tilt (the direction of slant): A neglected psychophysical variable. Perception \& Psychophysics, 33, 241-250.

Stevens, K. A., \& Brookes, A. (1987). Probing depth in monocular images. Biological Cybernetics, 56, 355-366.

TODD, J. T., \& REICHEL, F. T. (1989). Ordinal structure in the visual perception and cognition of smoothly curved surfaces. Psychological Review, 96, 643-657.

Yuille, A. L. (1989). Zero crossings on lines of curvature. Computer Vision, Graphics \& Image Processing, 45, 68-87.

\section{APPENDIX}

\section{The Surface}

The surface was the combination of four Gaussian hills, a different hill for each quadrant. This was obtained by the equation

$$
f(x, y)=2.5 e^{-\left(a x^{2}+b y^{2}+0.4 x y\right) / 2.0}
$$

where

$$
\begin{aligned}
& \text { if } x>0 \text { and } y>0 \text { then } a=0.9 \text { and } b=0.8 \text { (Quadrant 1) } \\
& \text { if } x<0 \text { and } y>0 \text { then } a=0.7 \text { and } b=0.8 \text { (Quadrant 2) } \\
& \text { if } x<0 \text { and } y<0 \text { then } a=0.7 \text { and } b=0.5 \text { (Quadrant 3) } \\
& \text { if } x>0 \text { and } y<0 \text { then } a=0.9 \text { and } b=0.5 \text { (Quadrant 4) }
\end{aligned}
$$

To obtain its final orientation, this surface was then rotated $22.5^{\circ}$ clockwise about the horizontal.

(Manuscript received July 21, 1994; revision accepted for publication October 4, 1995.) 